\title{
Massive and massless higher spinning particles in odd dimensions
}

\author{
Fiorenzo Bastianelli, ${ }^{a}$ Roberto Bonezzi, ${ }^{a}$ Olindo Corradini ${ }^{b, c}$ and Emanuele Latini ${ }^{d}$ \\ ${ }^{a}$ Dipartimento di Fisica ed Astronomia, Università di Bologna and \\ INFN, Sezione di Bologna, \\ via Irnerio 46, I-40126 Bologna, Italy \\ ${ }^{b}$ Facultad de Ciencias en Física y Matemáticas, Universidad Autónoma de Chiapas, \\ Ciudad Universitaria, Tuxtla Gutiérrez 29050, México \\ ${ }^{c}$ Dipartimento di Scienze Fisiche, Informatiche e Matematiche, \\ Università di Modena e Reggio Emilia, \\ Via Campi 213/A, I-41125 Modena, Italy \\ ${ }^{d}$ Institut für Mathematik, Universität Zürich-Irchel, \\ Winterthurerstrasse 190, CH-8057 Zürich, Switzerland \\ E-mail: bastianelli@bo.infn.it, bonezzi@bo.infn.it, \\ olindo.corradini@unach.mx, emanuele.latini@math.uzh.ch
}

ABSTRACT: We study actions for massive bosonic particles of higher spins by dimensionally reducing an action for massless particles. For the latter we take a model with a $\mathrm{SO}(N)$ extended local supersymmetry on the worldline, that is known to describe massless (conformal) particles of higher spins in flat spacetimes of even dimensions. Dimensional reduction produces an action for massive spinning particles in odd dimensions. The field equations that emerge in a quantization à la Dirac are shown to be equivalent to the Fierz-Pauli ones. The massless limit generates a multiplet of massless states with higher spins, whose first quantized field equations have a geometric form with fields belonging to various types of Young tableaux. These geometric equations can be partially integrated to show their equivalence with the standard Fronsdal-Labastida equations. We covariantize our model to check whether an extension to curved spacetimes can be achieved. Restricting to (A)dS spaces, we find that the worldline gauge algebra becomes nonlinear, but remains first class. This guarantees consistency on such backgrounds. A light cone analysis confirms the presence of the expected propagating degrees of freedom. A covariant analysis is worked out explicitly for the massive case, which is seen to give rise to the Fierz-Pauli equations extended to (A)dS spaces. It is worth noting that in $D=3$ the massless limit of our model with $N \rightarrow \infty$ has the same field content of the Vasiliev's theory that accommodates each spin exactly once.

Keywords: Higher Spin Gravity, Extended Supersymmetry, Sigma Models

ArXiv EPRINT: 1407.4950 


\section{Contents}

1 Introduction 1

2 Review of the $\mathrm{SO}(\mathrm{N})$ massless spinning particles 2

3 Dimensional reduction, massive particles, and massless limit 7

$\begin{array}{lll}3.1 & \text { Light cone analysis } & 8\end{array}$

$\begin{array}{ll}3.2 & \text { Covariant analysis }\end{array}$

$\begin{array}{ll}\text { 3.2.1 The massive case: Pauli-Fierz } & 12\end{array}$

3.2.2 The massless limit: Fronsdal-Labastida multiplets 14

$\begin{array}{ll}3.2 .3 \text { Gauge invariance } & 16\end{array}$

4 Coupling to curved space: (A)dS manifolds $\quad 17$

$\begin{array}{lll}4.1 & \text { Light cone analysis } & 19\end{array}$

$\begin{array}{lll}4.2 & \text { Covariant analysis: massive case } & 20\end{array}$

$\begin{array}{ll}4.3 \text { Massless limit: an example } & 21\end{array}$

5 Conclusions 24

\section{Introduction}

Higher spin field theories have recently been the focus of much interest. One of the main motivations arises from the study of the interacting higher spin field equations found by Vasiliev [1-3]. They involve an infinite number of higher spin fields on AdS spaces, and find interesting applications in AdS/CFT dualities [4-10]. For an introduction to these subjects see, for example, the reviews [11-15] and references therein.

One approach to study free higher spin fields in flat and curved backgrounds is to analyze the first quantization of relativistic particles. This approach was followed in [1618 ], which focused on massless spinning particles in even spacetime dimensions. In those references a certain class of higher spin states, defined by the $\mathrm{SO}(N)$ spinning particle action of [19-21], was analyzed in great details. The covariant quantization of the spinning particles was analyzed in flat and (A)dS spaces to show how well-known higher spin (HS) field equations would emerge from the Dirac quantization procedure. In addition, the path integral quantization was used to give a worldline representation of the one-loop effective action on (A)dS spaces, allowing for the calculation of the heat kernel coefficients corresponding to the divergencies of the effective action in $D=4$.

The standard $\mathrm{SO}(N)$ spinning particle action describes massless (in fact, conformal [22, 23]) particles of higher spin in spacetimes of even dimensions. They can be coupled to conformally flat backgrounds [17], which include in particular (A)dS spaces [24]. However, 
in odd dimensions the model is empty. The gauging of the full $\mathrm{SO}(N)$ extended worldline supersymmetry, which is the defining property of the model, constrains the propagating degrees of freedom to be that of a pure massless particle of spin $s=\frac{N}{2}$, but forces at the same time the spacetime dimension to be even dimensional (this happens for $N>2$ i.e. $s>1$ ). One could gauge a subgroup of the $\mathrm{SO}(N)$ symmetry group to describe a multiplet of spinning particles, a fact which is even desirable within the prospect of introducing interactions, but that would prevent an extension to curved backgrounds, as the gauging of the $\mathrm{SO}(N)$ charges is instrumental in providing a first class algebra on curved spaces [17].

Here we wish to continue the analysis of HS fields within the worldline approach and set ourselves to study bosonic massive and massless higher spinning particles in odd dimensions. We consider the introduction of a mass by dimensional reduction of the $\mathrm{SO}(N)$ spinning particle, and provide some solutions to the problems mentioned previously. By construction, the emerging model has massive degrees of freedom in odd dimensions only. Taking the massless limit gives a multiplet of HS particles in odd dimensions. The analysis of the physical degrees of freedom carried by the particle is performed both through a light cone approach and through a covariant approach. The latter is accomplished by using the Dirac quantization method. It shows how the dynamics is described in a gauge invariant way through linearized curvatures (which may be expressed in terms of gauge potentials if desired). Having performed the analysis in flat space, we proceed by noting that the gauge algebra can be covariantized to include (A)dS backgrounds, while keeping it first class. This provides a consistent model for both the massive and massless cases on (A)dS spaces. We perform again a light cone analysis to confirm that the particle propagates the same degrees of freedom as in flat space. A covariant analysis is carried out explicitly for the massive case, and we find that the covariant Dirac constraints can be reduced to the Fierz-Pauli equations extended to (A)dS spaces. A similar treatment of the massless case is more complex, and we content ourself to report the explicit example of $s=2$ in $D=3$. It is interesting to note that in the massless case and for integer $s=\frac{N}{2} \rightarrow \infty$ one finds the same field content appearing in the three dimensional Vasiliev's theory, where each integer spin occurs precisely once. The coupling to (A)dS is presumably crucial for having a chance of studying the interactions carried by the Vasiliev's model in a first quantized approach.

\section{Review of the $\mathrm{SO}(\mathrm{N})$ massless spinning particles}

We start reviewing the action of the $\mathrm{SO}(N)$ massless spinning particle, that describes a particle of spin $s=\frac{N}{2}$ upon quantization. This is mainly to introduce notations, conventions and methods. We consider the motion on a flat $(D+1)$-dimensional Minkowski space $M_{D+1}$ and take $N$ even to restrict ourselves to bosonic particles of integer spin $s$. The dynamical variables of the spinning particle are given by:

- the cartesian coordinates $x^{m}$ of the particle on $M_{D+1}(m=0,1, \ldots, D)$

- their conjugate momenta $p_{m}$

- $N$ real Grassmann variables with spacetime vector indices $\psi_{i}^{m}(i=1, \ldots, N)$ 
- the $\mathrm{SO}(N)$-extended supergravity multiplet on the worldline, whose gauge fields are made up by the einbein $e$, the $N$ gravitinos $\chi_{i}$, and the $\mathrm{SO}(N)$ gauge field $a_{i j}$.

The phase space action of the model, $S=\int d \tau L$, is identified by the lagrangian ${ }^{1}$

$$
L=p_{m} \dot{x}^{m}+\frac{i}{2} \psi_{i m} \dot{\psi}_{i}^{m}-e \underbrace{\left(\frac{1}{2} p_{m} p^{m}\right)}_{\mathcal{H}}-i \chi_{i} \underbrace{\left(p_{m} \psi_{i}^{m}\right)}_{\mathcal{Q}_{i}}-\frac{1}{2} a_{i j} \underbrace{\left(i \psi_{i}^{m} \psi_{j m}\right)}_{\mathcal{J}_{i j}}
$$

where $\mathcal{H}, \mathcal{Q}_{i}, \mathcal{J}_{i j}$ denote first class constraints gauged by $e, \chi_{i}, a_{i j}$. The kinetic term defines the phase space symplectic structure and fixes the graded Poisson brackets

$$
\left\{x^{m}, p_{n}\right\}_{P B}=\delta_{n}^{m}, \quad\left\{\psi_{i}^{m}, \psi_{j}^{n}\right\}_{P B}=-i \eta^{m n} \delta_{i j}
$$

(other independent brackets vanish). With them one computes the constraint algebra

$$
\begin{aligned}
\left\{\mathcal{Q}_{i}, \mathcal{Q}_{j}\right\}_{P B} & =-2 i \delta_{i j} \mathcal{H} \\
\left\{\mathcal{J}_{i j}, \mathcal{Q}_{k}\right\}_{P B} & =\delta_{j k} \mathcal{Q}_{i}-\delta_{i k} \mathcal{Q}_{j} \\
\left\{\mathcal{J}_{i j}, \mathcal{J}_{k l}\right\}_{P B} & =\delta_{j k} \mathcal{J}_{i l}-\delta_{i k} \mathcal{J}_{j l}-\delta_{j l} \mathcal{J}_{i k}+\delta_{i l} \mathcal{J}_{j k}
\end{aligned}
$$

which is first class. This algebra is known as the $\mathrm{SO}(N)$-extended susy algebra in one dimension, as it contains $N$ real susy charges $\mathcal{Q}_{i}$. They transform in the vector representation of $\mathrm{SO}(N)$ (the so-called R-symmetry group) generated by the $\mathcal{J}_{i j}$ charges, and close on the Hamiltonian $\mathcal{H}$.

In a quantization à la Dirac, the constraint functions $C^{A}:=\left(\mathcal{H}, \mathcal{Q}_{i}, \mathcal{J}_{i j}\right)$ become operators that produce the massless higher spin (HS) field equations. One may write them as $C^{A}|R\rangle=0$, where $|R\rangle$ is a vector of the extended Hilbert space. The solutions of these constraint equations make up the subspace of physical states. The remaining Schrödinger equation implies that the physical fields do not depend on the time parameter $\tau$. These constraint equations have the property of being conformally invariant [22, 23], and take the form of the Bargmann-Wigner equations [25], studied in arbitrary spacetime dimensions in $[26,27]$. Let us describe them. The physical states $|R\rangle$ are contained in a tensor

$$
R_{m_{1}^{1} \ldots m_{d}^{1}, \ldots, m_{1}^{s} \ldots m_{d}^{s}}
$$

with $s$ blocks of $d=\frac{D+1}{2}$ antisymmetric indices ${ }^{2}$ that satisfies the properties:

(i) it is completely traceless and has the symmetries of a Young tableau with $d$ rows and $s$ columns (this follows from the $\mathcal{J}$ constraints)

$$
R_{m_{1}^{1} \ldots m_{d}^{1}, \ldots, m_{1}^{s} \ldots m_{d}^{s}} \sim d\left\{\begin{array}{l|l|l|l|}
\hline & & & \\
\hline & & & \\
\hline & & & \\
\hline
\end{array}\right.
$$

\footnotetext{
${ }^{1}$ The Minkowski metric $\eta_{m n} \sim(-,+, \cdots,+)$ is used to raise and lower spacetime indices. Indices named $m, n, \ldots$ etc. refer to spacetime indices $(m, n=0,1, \ldots D)$, while those named $i, j, \ldots$ etc. stand for internal $\mathrm{SO}(N)$ indices $(i, j=1, \ldots, N)$.

${ }^{2}$ We separate different blocks of antisymmetric indices by commas; $D+1$ must be even for nontrivial solutions so that $d=\frac{D+1}{2}$ is integer.
} 
(ii) it satisfies integrability conditions (from half of the $\mathcal{Q}$ constraints)

$$
\partial_{[m} R_{\left.m_{1}^{1} \ldots m_{d}^{1}\right], \ldots, m_{1}^{s} \ldots m_{d}^{s}}=0
$$

interpreted as Bianchi identities once solved,

(iii) it satisfies Maxwell equations (from the other half of the $\mathcal{Q}$ constraints)

$$
\partial^{m} R_{m m_{2}^{1} \ldots m_{d}^{1}, \ldots, m_{1}^{s} \ldots m_{d}^{s}}=0 .
$$

The $\mathcal{H}$ constraint is automatically satisfied as consequence of the constraint algebra. These are geometric equations for free conformal fields of integer spin $s$, equivalent to the massless Bargmann-Wigner equations. They are called geometric as the tensors $R$ can be interpreted as (linearized) curvatures, as we are going to show later on.

To derive these equations it is useful to take complex combinations of the $N=2 \mathrm{~s}$ indices and define (for $I=i=1, \ldots, s$ )

$$
\psi_{I}=\frac{1}{\sqrt{2}}\left(\psi_{i}+i \psi_{i+s}\right), \quad \bar{\psi}^{I}=\frac{1}{\sqrt{2}}\left(\psi_{i}-i \psi_{i+s}\right) .
$$

Their non trivial quantum anticommutators are given by

$$
\left\{\psi_{I}^{m}, \bar{\psi}^{J n}\right\}=\eta^{m n} \delta_{I}^{J}
$$

and describe a set of fermionic creation/annihilation operators. In this basis only the subgroup $\mathrm{U}(s) \subset \mathrm{SO}(2 s)$ is manifest. The susy charges take the form $\mathcal{Q}_{I}=\psi_{I}^{m} p_{m}$ and $\overline{\mathcal{Q}}^{I}=\bar{\psi}^{I m} p_{m}$, and the susy algebra breaks up into

$$
\left\{\mathcal{Q}_{I}, \overline{\mathcal{Q}}^{J}\right\}=2 \delta_{I}^{J} \mathcal{H}, \quad\left\{\mathcal{Q}_{I}, \mathcal{Q}_{J}\right\}=\left\{\overline{\mathcal{Q}}^{I}, \overline{\mathcal{Q}}^{J}\right\}=0 .
$$

The $\mathrm{SO}(N)$ generators split as $\mathcal{J}_{i j} \sim\left(\mathcal{J}_{I \bar{J}}, \mathcal{J}_{I J}, \mathcal{J}_{\bar{I} \bar{J}}\right):=\left(\mathcal{J}_{I}{ }^{J}, \mathcal{K}_{I J}, \overline{\mathcal{K}}^{I J}\right)$, which we normalize as

$$
\mathcal{J}_{I}^{J}=\psi_{I} \cdot \bar{\psi}^{J}-d \delta_{I}^{J}, \quad \mathcal{K}_{I J}=\psi_{I} \cdot \psi_{J}, \quad \overline{\mathcal{K}}^{I J}=\bar{\psi}^{I} \cdot \bar{\psi}^{J}
$$

(note that $\mathcal{J}_{I}^{J}$ for fixed $I=J$ is a hermitian operator with real eigenvalues). Then, the $\mathrm{SO}(N)$ algebra breaks up into

$$
\begin{aligned}
& {\left[\mathcal{J}_{I}{ }^{J}, \mathcal{J}_{K}{ }^{L}\right]=\delta_{K}^{J} \mathcal{J}_{I}{ }^{L}-\delta_{I}^{L} \mathcal{J}_{K}{ }^{J}} \\
& {\left[\mathcal{J}_{I}{ }^{J}, \mathcal{K}_{K L}\right]=\delta_{K}^{J} \mathcal{K}_{I L}+\delta_{L}^{J} \mathcal{K}_{K I}} \\
& {\left[\mathcal{J}_{I}{ }^{J}, \overline{\mathcal{K}}^{K L}\right]=-\delta_{I}^{K} \overline{\mathcal{K}}^{J L}-\delta_{I}^{L} \overline{\mathcal{K}}^{K J}} \\
& {\left[\mathcal{K}_{I J}, \overline{\mathcal{K}}^{K L}\right]=\delta_{J}^{K} \mathcal{J}_{I}{ }^{L}-\delta_{J}^{L} \mathcal{J}_{I}{ }^{K}-\delta_{I}^{K} \mathcal{J}_{J}{ }^{L}+\delta_{I}^{L} \mathcal{J}_{J}{ }^{K}}
\end{aligned}
$$

with other commutators vanishing. The first line identifies the manifest $\mathrm{U}(s)$ subalgebra. Finally, the remaining non trivial part of the constraint algebra takes the form

$$
\begin{aligned}
& {\left[\mathcal{J}_{I}{ }^{J}, \mathcal{Q}_{K}\right]=\delta_{K}^{J} \mathcal{Q}_{I}} \\
& {\left[\mathcal{J}_{I}^{J}, \overline{\mathcal{Q}}^{K}\right]=-\delta_{I}^{K} \overline{\mathcal{Q}}^{J}} \\
& {\left[\overline{\mathcal{K}}^{I J}, \mathcal{Q}_{K}\right]=\delta_{K}^{J} \overline{\mathcal{Q}}^{I}-\delta_{K}^{I} \overline{\mathcal{Q}}^{J}} \\
& {\left[\mathcal{K}_{I J}, \overline{\mathcal{Q}}^{K}\right]=\delta_{J}^{K} \mathcal{Q}_{I}-\delta_{I}^{K} \mathcal{Q}_{J} .}
\end{aligned}
$$


We now analyze the constraints in a quantization à la Dirac. The fermionic operators can be treated using a basis of fermionic coherent states, so that they can be realized by letting $\psi_{I}^{m}$ act as multiplication by the Grassmann variables $\psi_{I}^{m}$, and $\bar{\psi}_{m}^{I}$ as derivation by the Grassmann variable $\psi_{I}^{m}$ (i.e. $\bar{\psi}_{m}^{I}=\frac{\partial}{\partial \psi_{I}^{m}}$; we refrain from denoting operators with a hat, as no confusion can arise). Using in addition the coordinate representation for the position and momentum operators, one may describe a generic state $|R\rangle$ of the full Hilbert space by the wave function

$$
R(x, \psi)=\left(\langle x|\otimes\langle\psi|) \mid R\rangle=\sum_{A_{i}=0}^{D+1} R_{m_{1} \ldots m_{A_{1}}, \ldots, n_{1} \ldots n_{A_{s}}}(x) \psi_{1}^{m_{1}} \ldots \psi_{1}^{m_{A_{1}}} \ldots \psi_{s}^{n_{1}} \ldots \psi_{s}^{n_{A_{s}}}\right.
$$

which contains all possible tensors with $s$ blocks of indices, completely antisymmetric in each block.

In the chosen representation the $\mathrm{SO}(N)$ generators take the form

$$
\mathcal{J}_{I}^{J}=\psi_{I} \cdot \frac{\partial}{\partial \psi_{J}}-d \delta_{I}^{J}, \quad \mathcal{K}_{I J}=\psi_{I} \cdot \psi_{J}, \quad \overline{\mathcal{K}}^{I J}=\frac{\partial}{\partial \psi_{I}} \cdot \frac{\partial}{\partial \psi_{J}} .
$$

The operator $\mathcal{J}_{I}{ }^{I}$ at fixed $I$ counts the number of fermions $\psi_{I}^{m}$ of flavor $I$ minus $d$ (this constant arises from a graded-symmetric quantum ordering prescription), while $\mathcal{J}_{I}^{J}$ removes from the wavefunction a fermion $\psi_{J}^{m}$ and replaces it with a fermion $\psi_{I}^{m}$ (the fermions of each species antisymmetrize the corresponding indices of the tensor that multiplies them). In addition, $\mathcal{K}_{I J}=\psi_{I} \cdot \psi_{J}=\psi_{I}^{m} \eta_{m n} \psi_{J}^{n}$ acts on the various tensors by adding one index in the $I$-th block and one index the $J$-th block by multiplying with the metric tensor $\eta_{m n}$, each block being then automatically antisymmetrized. Similarly, $\overline{\mathcal{K}}^{I J}=\frac{\partial}{\partial \psi_{I}} \cdot \frac{\partial}{\partial \psi_{J}}=\frac{\partial}{\partial \psi_{I}^{m}} \eta^{m n} \frac{\partial}{\partial \psi_{J}^{n}}$ computes traces by contracting one index of the $I$-th block with one index the $J$-th block through the metric tensor. Then, it is easy to see that the corresponding constraints imply

$$
\begin{aligned}
\mathcal{J}_{I}^{I}|R\rangle=0(I \text { fixed }) & \Rightarrow R=R_{m_{1} \ldots m_{d}, \ldots, n_{1} \ldots n_{d}}(x) \psi_{1}^{m_{1}} \ldots \psi_{1}^{m_{d}} \ldots \psi_{s}^{n_{1}} \ldots \psi_{s}^{n_{d}} \\
\mathcal{J}_{I}{ }^{J}|R\rangle=0(I \neq J) & \Rightarrow R \text { satisfies algebraic Bianchi identities } \\
\overline{\mathcal{K}}^{I J}|R\rangle=0 & \Rightarrow R \text { traceless } \\
\mathcal{K}_{I J}|R\rangle=0 & \Rightarrow R \text { traceless } .
\end{aligned}
$$

Similarly, the constraints $Q_{i}=\left(\mathcal{Q}_{I}, \bar{Q}^{I}\right)$ produce

$$
\begin{aligned}
& \mathcal{Q}_{I}|R\rangle=0 \quad \Rightarrow \quad R \text { closed (integrability conditions } \rightarrow \text { potentials) } \\
& \overline{\mathcal{Q}}^{I}|R\rangle=0 \quad \Rightarrow \quad R \text { co-closed (Maxwell equations) }
\end{aligned}
$$

The constraint $\mathcal{H}$ is automatically satisfied as a consequence of the algebra.

Note that the constraints (2.16) and (2.17) correspond to the generators of the manifest $\mathrm{U}(s) \subset \mathrm{SO}(2 s)$. The tensor $R$ solving these equations has $s$ blocks with $d$ antisymmetric indices each, consequence of (2.16), and satisfies algebraic Bianchi identities of the form

$$
R_{\left[m_{1} \ldots m_{d}, n_{1}\right] \ldots n_{d}, \ldots}=0
$$


where $[\ldots]$ indicates antisymmetrization, consequence of (2.17). There is also a symmetry under an exchange of the blocks. It can be proved by using finite $\mathrm{SO}(s) \subset \mathrm{U}(s)$ rotations. For example, a $\frac{\pi}{2}$ rotation in the $I-J$ plane, that implements $\psi_{I} \rightarrow \psi_{J}$ and $\psi_{J} \rightarrow-\psi_{I}$, implies symmetry under the exchange of block $I$ with block $J$. Note that the fermionic Fock vacuum $|\Omega\rangle \sim \Omega(x)$ is not invariant under $[\mathrm{U}(1)]^{s} \subset \mathrm{U}(s)$, as all generators $\mathcal{J}_{I}^{I}$ with fixed $I$ transform it by an infinitesimal phase $\left(\mathcal{J}_{I}^{I}|\Omega\rangle=-d|\Omega\rangle\right)$. It is the vector $|R\rangle$ in eq. (2.16) that is invariant. Summarizing, the constraints $\mathcal{J}_{I}^{J}$, i.e. those belonging to $\mathrm{U}(s)$, select an irreducible representation of the general linear group $\mathrm{GL}(D+1)$ identified by a Young tableau with $d=\frac{D+1}{2}$ rows and $s=\frac{N}{2}$ columns, as depicted in (2.5).

The constraint $\overline{\mathcal{K}}^{I J}$ removes all possible traces from this tensor, and produces an irreducible representation of the Lorentz group $\mathrm{SO}(D, 1)$. The constraints due to $\mathcal{K}_{I J}$ do not give new independent relations: they say that pieces equivalent to pure traces must vanish. The equivalence of $\mathcal{K}_{I J}$ and $\overline{\mathcal{K}}^{I J}$ constraints is not a consequence of the algebra, but can be viewed as a consequence of a duality symmetry enjoyed by the spinning particle. Indeed, one can realize the Hodge operator acting in the $I$-th block by

$$
\star_{I}: \psi_{I} \leftrightarrow \bar{\psi}^{I}, \quad\left(\star_{I}\right)^{2}=1 .
$$

The exchange $\psi_{I} \leftrightarrow \bar{\psi}^{I}$ maps the lowest state (in the fermionic Fock vacuum) with the highest state, and so on, and it is seen to correspond to a dualization of the antisymmetric indices of the tensor $R$ belonging to the $I$-th block. It is obtained by a discrete $\mathrm{O}(N)$ symmetry transformation (that reflects one real $\psi_{i}$ fermion). Denote now $\star_{I J}=\star_{I} \star_{J}$ (this combined transformation can be done within $\mathrm{SO}(N))$. Then

$$
\mathcal{K}_{I J}|R\rangle=0 \quad \Rightarrow \quad\left(\star_{I J} \mathcal{K}_{I J} \star_{I J}\right)\left(\star_{I J}|R\rangle\right)=\overline{\mathcal{K}}^{I J}\left|R^{\left(\star_{I J}\right)}\right\rangle=0
$$

which implies that $R^{\left(\star_{I J}\right)}$ is traceless when contracting an index of the $I$-th block with an index of the $J$-th block. By $R^{\left(\star_{I J}\right)}$ we indicate the tensor dual to $R$ in both set of indices, those of the block $I$ and those of the block $J$. Using $\epsilon \epsilon \sim \delta \ldots \delta$, one may check that tracelessness of $R^{\left(\star_{I J}\right)}$ implies tracelessness of $R$ as well. Finally, note that the $\overline{\mathcal{Q}}^{I}$ constraint is a consequence of (2.20) and (2.18), since the $\left[\overline{\mathcal{K}}^{I J}, \mathcal{Q}_{K}\right]=\delta_{K}^{J} \overline{\mathcal{Q}}^{I}-\delta_{K}^{I} \overline{\mathcal{Q}}^{J}$.

We have verified that an independent set of constraints is given by $\left(\mathcal{J}_{I}^{J}, \mathcal{Q}_{I}, \overline{\mathcal{K}}^{I J}\right)$. They can be implemented in that order to make contact with the Fonsdal-Labastida formulation of higher spin fields (with or without compensators) for the particular spin representations carried by the $\mathrm{SO}(N)$ particle. Let us review these last steps as well. Gauge potentials $|\phi\rangle$ can be introduced by integrating the $\mathcal{Q}_{I}$ constraint as

$$
|R\rangle=q|\phi\rangle
$$

where $q=\mathcal{Q}_{1} \mathcal{Q}_{2} \ldots \mathcal{Q}_{s}$. This follows from the nilpotency of the $\mathcal{Q}_{I}$ 's together with a Poincaré lemma stating that the related cohomologies are trivial in Minkowski space (all closed forms are exact). Then, the constraints $\mathcal{J}_{I}^{J}$ are implemented by taking $|\phi\rangle$ to satisfy

$$
\mathcal{J}_{I}^{J}|\phi\rangle=-\delta_{I}{ }^{J}|\phi\rangle
$$

that fixes $|\phi\rangle$ to contain an irreducible tensor under $\operatorname{GL}(D+1)$ with Young tableau of the form 


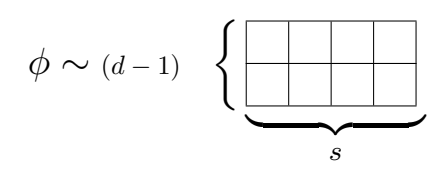

Finally, the remaining constraints $\overline{\mathcal{K}}^{I J}$ (the trace constraints) implement the dynamical equations. One computes

$$
\overline{\mathcal{K}}^{I J}|R\rangle=\overline{\mathcal{K}}^{I J} q|\phi\rangle=q^{I J} \underbrace{\left[-2 \mathcal{H}+\mathcal{Q}_{I} \overline{\mathcal{Q}}^{I}+\frac{1}{2} \mathcal{Q}_{I} \mathcal{Q}_{J} \overline{\mathcal{K}}^{I J}\right]}_{\mathcal{G}}|\phi\rangle=0
$$

where $q^{I J}:=\frac{\partial}{\mathcal{Q}_{I}} \frac{\partial}{\mathcal{Q}_{J}} q$ and $\mathcal{G}$ is the Fronsdal-Labastida operator ${ }^{3}$ which is manifestly $\mathrm{U}(s)$ invariant (one checks that $\left[\mathcal{J}_{I}{ }^{J}, \mathcal{G}\right]=0$ ). The product of $s+1 \mathcal{Q}_{I}$ 's must vanish, so that one may partially integrate this last equation to obtain the Fronsdal-Labastida equation with compensators

$$
\mathcal{G}|\phi\rangle=\mathcal{Q}_{I} \mathcal{Q}_{J} \mathcal{Q}_{K}\left|\rho^{I J K}\right\rangle
$$

where the right hand side parametrizes an element of the kernel of $q^{I J}$, and the compensator $\left|\rho^{I J K}\right\rangle$ has a Young tableau of $G L(D+1)$ of the form

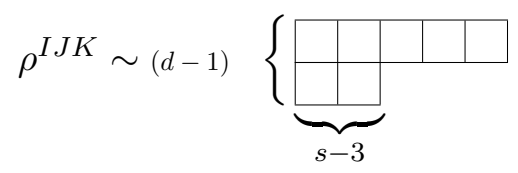

The gauge symmetries of the Fronsdal-Labastida equation with compensators are given by

$$
\delta|\phi\rangle=\mathcal{Q}_{I}\left|\xi^{I}\right\rangle, \quad \delta\left|\rho^{I J K}\right\rangle=\frac{1}{2} \overline{\mathcal{K}}^{[I J}\left|\xi^{K]}\right\rangle .
$$

A partial gauge fixing can be used to set the compensators to vanish, and one is left with the original Fronsdal-Labastida equation

$$
\mathcal{G}|\phi\rangle=0
$$

with gauge symmetries generated by traceless gauge parameters. The use of compensators in this context was discussed in [30-32].

\section{Dimensional reduction, massive particles, and massless limit}

Massive spinning particles can be obtained by the Scherk-Schwarz mechanism [33] of dimensionally reducing the massless model on a flat spacetime of the form $M_{D} \times S^{1}$. In practice, one gauges the compact direction $x^{D}$, corresponding to $S^{1}$, by imposing the first

\footnotetext{
${ }^{3}$ It corresponds to the Fronsdal kinetic operator for higher spin fields in $D=4$ [28], extended to higher dimensions for generic tensors of the Lorentz group by Labastida [29].
} 
class constraint $p_{D}-m=0$. Setting $x^{m}=\left(x^{\mu}, x^{D}\right), p_{m}=\left(p_{\mu}, p_{D}\right)$, and $\psi_{i}^{m}=\left(\psi_{i}^{\mu}, \theta_{i}\right)$ one obtains in flat, odd $D$ dimensions

$$
\begin{aligned}
L= & p_{\mu} \dot{x}^{\mu}+\frac{i}{2} \psi_{i \mu} \dot{\psi}_{i}^{\mu}+\frac{i}{2} \theta_{i} \dot{\theta}_{i} \\
& -e \underbrace{\frac{1}{2}\left(p_{\mu} p^{\mu}+m^{2}\right)}_{\mathcal{H}}-i \chi_{i} \underbrace{\left(p_{\mu} \psi_{i}^{\mu}+m \theta_{i}\right)}_{\mathcal{Q}_{i}}-\frac{1}{2} a_{i j} \underbrace{\left(i \psi_{i}^{\mu} \psi_{j \mu}+i \theta_{i} \theta_{j}\right)}_{\mathcal{J}_{i j}} .
\end{aligned}
$$

The constraints satisfy again the same algebra written in (2.3), where $\mathrm{SO}(N)$ is manifest. As shown before, this algebra can be equivalently written as in (2.16)-(2.19) and (2.20)(2.21), where only the group $\mathrm{U}(s)$ is manifest. The latter form is useful to analyze and solve the quantum constraints.

\subsection{Light cone analysis}

Before discussing the covariant treatment of the constraints at the quantum level, let us present a light cone analysis to calculate and check the number of propagating physical degrees of freedom.

We define light cone coordinates by $x^{\mu}=\left(x^{+}, x^{-}, x^{a}\right)$ with $x^{ \pm}=\left(x^{D-1} \pm x^{0}\right) / \sqrt{2}$ and $x^{a}$ the transverse directions, so that $d s^{2}=2 d x^{+} d x^{-}+d x^{a} d x^{a}$. Note that vectors have light cone indices such that $p^{+}=p_{-}$and $p^{-}=p_{+}$.

One can set $x^{+}=\tau$ as gauge fixing condition, dual to the mass shell constraint $\mathcal{H}=0$. The gauge is well-fixed, as $\left\{x^{+}, p^{2}+m^{2}\right\}_{P B}=2 p_{-} \neq 0$ (recall that $p_{-}=p^{+}$is assumed to be invertible in light cone analysis). The constraint $p^{2}+m^{2}=0$ is then solved in terms of $p_{+}=-\frac{1}{2 p_{-}}\left(p_{T}^{2}+m^{2}\right)$, where $p_{T}^{2}=p^{a} p^{a}$ is the transverse momentum squared. The conjugate variables $\left(x^{+}, p_{+}\right)$of the phase space are thus eliminated. The parameter $x^{+}$is taken as the time parameter, and $-p_{+}=\frac{1}{2 p_{-}}\left(p_{T}^{2}+m^{2}\right)$ is the corresponding hamiltonian.

Then, one can gauge fix the Majorana fermions $\psi_{i}^{+}=0$. The local susy transformations act on the Majorana fermions as $\delta \psi_{i}^{m}=\left\{\psi_{i}^{m}, \epsilon^{j} \mathcal{Q}_{j}\right\}_{P B}=i \epsilon_{i} p^{m}$, so that using the infinitesimal transformations $\delta \psi_{i}^{+}=i \epsilon_{i} p^{+}$(which are non vanishing) one can set $\psi_{i}^{+}=0$. The gauge is well-fixed, and indeed $\left\{\psi_{i}^{+}, \mathcal{Q}_{j}\right\}=-i p^{+} \delta_{i j} \neq 0$. One may solve the constraints $\mathcal{Q}_{i}=0$ by setting $\psi_{i}^{-}=-\frac{1}{p_{-}}\left(p_{a} \psi_{i}^{a}+m \theta_{i}\right)$, and the conjugated variables $\left(\psi_{i}^{+}, \psi_{i}^{-}\right)$ are eliminated as independent phase space coordinates. The coordinates of the reduced phase space are now given by $\left(x^{-}, p_{-}\right),\left(x^{a}, p^{a}\right)$, and $\psi_{i}^{a}$, with lagrangian

$$
\begin{aligned}
L= & p_{-} \dot{x}^{-}+p_{a} \dot{x}^{a}+\frac{i}{2} \psi_{i a} \dot{\psi}_{i}^{a}+\frac{i}{2} \theta_{i} \dot{\theta}_{i}-\frac{1}{2 p_{-}}\left(p_{T}^{2}+m^{2}\right) \\
& -\frac{1}{2} a_{i j} \underbrace{\left(i \psi_{i}^{a} \psi_{j a}+i \theta_{i} \theta_{j}\right)}_{\mathcal{J}_{i j}} .
\end{aligned}
$$

One may try to reduce the phase space further, implementing the last constraint $\mathcal{J}_{i j}$. However, this can be done in a simpler way à la Dirac, since it produces purely algebraic constraints. This implementation proceeds as described previously, when discussing the massless case in $D+1$ even dimensions. Taking into account the unique quantum ordering 
of the quantum constraints $\mathcal{J}_{i j}$, one finds a sum of irreps of the $\mathrm{SO}(D-2)$ group that fill an irrep of the $\mathrm{SO}(D-1)$ rotation group corresponding precisely to the polarizations of a massive spin $s$ in $D$ dimensions (in higher dimensions by spin $s$ we mean a multiplet corresponding to a rectangular Young tableau with $s$ columns and $\frac{D-1}{2}$ rows). The corresponding degrees of freedom are counted by using a "factor over hook" type of formula, and their number is given by

$$
\operatorname{Dof}(D, s)=\frac{Y_{t}}{Y_{h}}
$$

where

$$
Y_{t}=\prod_{i=1}^{d-1} \frac{(s+i-2) !(2 s+2 i-2) !}{(2 i-2) !(2 s+i-2) !}, \quad Y_{h}=\prod_{i=1}^{d-1} \frac{(s+i-1) !}{(i-1) !}, \quad d:=\frac{D+1}{2}
$$

In particular, in $D=3$ one finds two degrees of freedom for any spin $s>0$. Of course, this is identical to the polarizations of a massless spin $s$ particle in one dimension higher, a fact that is rather evident from the dimensional reduction process.

\subsection{Covariant analysis}

We are now ready to give a covariant analysis, implementing the constraints of the Dirac quantization scheme. We can partially solve them to make contact with known relativistic higher spin wave equations, that is Fierz-Pauli in the massive case and Fronsdal-Labastida in the massless one. This analysis is done intrinsically, i.e. working directly in $D$ dimension, without considering the dimensional reduction. This is the strategy that we follow once we extend the model to (A)dS backgrounds. Of course, keeping in mind the dimensional reduction simplifies a bit the derivation of the field equations in flat space.

Thus, we work in odd $D$ dimensional flat spacetime, with $D=2 d-1, d \geq 2$, and use the complex $\mathrm{U}(s)$ covariant combinations for fermions as defined in section 2 , that now read $\left(\psi_{I}^{\mu}, \bar{\psi}^{\mu I}\right)$ and $\left(\theta_{I}, \bar{\theta}^{I}\right)$. As before, we represent $\psi$ 's and $\theta$ 's as multiplications by the corresponding Grassmann variable, and $\bar{\psi}$ 's, $\bar{\theta}$ 's as derivatives thereof. We indicate a generic state of the model by $|\mathcal{R}\rangle$, and we mostly work with the wave function $\mathcal{R}(x, \psi, \theta)=$ $\left(\langle x| \otimes\langle\psi|\otimes\langle\theta|) \mid \mathcal{R}\rangle\right.$, which has a finite Taylor expansion in $\psi_{I}^{\mu}$ and $\theta_{I}$, where $I=1, \ldots, s$. Since the $\theta$ content will distinguish between different types of spacetime tensors, we find it convenient to isolate it explicitly writing the state as

$$
\mathcal{R}(x, \psi, \theta)=\sum_{n=0}^{s} \frac{1}{n !} R^{I_{1} \ldots I_{n}}(x, \psi) \theta_{I_{1}} \ldots \theta_{I_{n}},
$$

with $R^{I_{1} \ldots I_{n}}:=R^{\left[I_{1} \ldots I_{n}\right]}$ being totally antisymmetric in the $I$ indices. Here and in what follows [...] will always denote weighted antisymmetrization. In the constraints the $\left(\psi, \partial_{\psi}\right)$ and $\left(\theta, \partial_{\theta}\right)$ parts play different roles: the first performs algebraic operations on the single tensors contained in $R^{I_{1} \ldots I_{n}}$, while the second mixes different tensor structures. For this 
reason we write the constraints in split form as follows

$$
\begin{aligned}
\mathcal{J}_{I}^{J} & =\psi_{I}^{\mu} \frac{\partial}{\partial \psi_{J}^{\mu}}+\theta_{I} \frac{\partial}{\partial \theta_{J}}-d \delta_{I}^{J}=J_{I}^{J}+\theta_{I} \frac{\partial}{\partial \theta_{J}}-d \delta_{I}^{J}, \\
\mathcal{K}_{I J} & =\psi_{I}^{\mu} \psi_{J \mu}+\theta_{I} \theta_{J}=g_{I J}+\theta_{I} \theta_{J} \\
\overline{\mathcal{K}}^{I J} & =\frac{\partial^{2}}{\partial \psi_{I}^{\mu} \partial \psi_{J} \mu}+\frac{\partial^{2}}{\partial \theta_{I} \partial \theta_{J}}=\operatorname{tr}^{I J}+\frac{\partial^{2}}{\partial \theta_{I} \partial \theta_{J}}
\end{aligned}
$$

for the $\mathrm{SO}(2 s)$ algebra operators, and

$$
\begin{aligned}
\mathcal{Q}_{I} & =\psi_{I}^{\mu} p_{\mu}+m \theta_{I}=Q_{I}+m \theta_{I}, \quad \overline{\mathcal{Q}}^{I}=p_{\mu} \frac{\partial}{\partial \psi_{I}^{\mu}}+m \frac{\partial}{\partial \theta_{I}}=\bar{Q}^{I}+m \frac{\partial}{\partial \theta_{I}} \\
\mathcal{H} & =\frac{1}{2}\left(p^{2}+m^{2}\right)=H+\frac{m^{2}}{2}
\end{aligned}
$$

for the supersymmetry part. We named $g_{I J}:=\psi_{I}^{\mu} \psi_{J \mu}$ and $\operatorname{tr}^{I J}:=\frac{\partial^{2}}{\partial \psi_{I}^{\mu} \partial \psi_{J} \mu}$ the $D$ dimensional parts of $\mathcal{K}_{I J}$ and $\overline{\mathcal{K}}^{I J}$ in order to emphasize their algebraic meaning. The algebra of the $D$-dimensional operators $J_{I}^{J}, g_{I J}, \operatorname{tr}^{I J}, Q_{I}, \bar{Q}^{I}$ and $H$ is the same as the massless algebra presented in section 2 in $D+1$ dimensions, up to the normal ordering constant that for simplicity we have not included in $J_{I}^{J}:=\psi_{I}^{\mu} \frac{\partial}{\partial \psi_{J}^{\mu}}$, but we give it here for completeness. The $\mathrm{SO}(N)$ part reads

$$
\begin{aligned}
{\left[J_{I}^{J}, J_{K}{ }^{L}\right] } & =\delta_{K}^{J} J_{I}^{L}-\delta_{I}^{L} J_{K}^{J} \\
{\left[J_{I}^{J}, g_{K L}\right] } & =\delta_{K}^{J} g_{I L}+\delta_{L}^{J} g_{K I} \\
{\left[J_{I}^{J}, \operatorname{tr}^{K L}\right] } & =-\delta_{I}^{K} \operatorname{tr}^{J L}-\delta_{I}^{L} \operatorname{tr}^{K J} \\
{\left[g_{I J}, \operatorname{tr}^{K L}\right] } & =4 \delta_{[J}^{[k} J_{I]}^{L]}-(2 d-1)\left(\delta_{J}^{K} \delta_{I}^{L}-\delta_{I}^{K} \delta_{J}^{L}\right)
\end{aligned}
$$

while the $R$-symmetry rotations are given by

$$
\begin{aligned}
{\left[J_{I}{ }^{J}, Q_{K}\right] } & =\delta_{K}^{J} Q_{I} \\
{\left[J_{I}^{J}, \bar{Q}^{K}\right] } & =-\delta_{I}^{K} \bar{Q}^{J} \\
{\left[\operatorname{tr}^{I J}, Q_{K}\right] } & =\delta_{K}^{J} \bar{Q}^{I}-\delta_{K}^{I} \bar{Q}^{J} \\
{\left[g_{I J}, \bar{Q}^{K}\right] } & =\delta_{J}^{K} Q_{I}-\delta_{I}^{K} Q_{J},
\end{aligned}
$$

and the susy algebra is

$$
\left\{Q_{I}, \bar{Q}^{J}\right\}=2 \delta_{I}^{J} H, \quad\left\{Q_{I}, Q_{J}\right\}=\left\{\bar{Q}^{I}, \bar{Q}^{J}\right\}=0
$$

To understand the tensor content of the various $R^{I_{1} \ldots I_{n}}$ terms we recall that the diagonal $\mathcal{J}$ 's are number operators that count the numbers $\mathrm{N}_{\psi_{I}}$ of $\psi_{I}$ 's and $\mathrm{N}_{\theta_{I}}$ of $\theta_{I}$ 's. The constraints $\mathcal{J}_{I}^{I} \mathcal{R}=0$, where $I$ is fixed and not summed, amount then to

$$
\left(\mathrm{N}_{\psi_{I}}+\mathrm{N}_{\theta_{I}}\right) \mathcal{R}=d \mathcal{R}
$$


This means that in $\mathcal{R}$ we have $d$ antisymmetric indices in the $I$-th group whenever the $\theta_{I}$ is not present, while we have $d-1$ antisymmetric indices when it is. From the decomposition (3.5), it is thus clear that $R^{I_{1} \ldots I_{n}}$ contains $n$ "short" columns ${ }^{4}$ of $(d-1)$ indices labeled by $I_{1} \ldots I_{n}$, and the remaining $(s-n)$ "long" columns of $d$ indices, i.e.

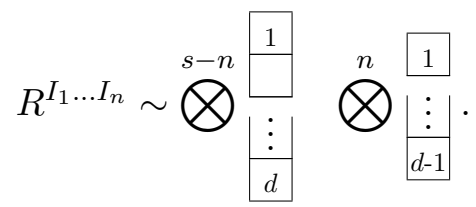

This is covariantly stated by splitting in $\theta$ the $\mathcal{J}_{I}^{J} \mathcal{R}=0$ equation, that reads

$$
\left(J_{K}^{L}-d \delta_{K}^{L}\right) R^{I_{1} \ldots I_{n}}+n(-)^{n-1} R^{L\left[I_{1} \ldots I_{n-1}\right.} \delta_{K}^{\left.I_{n}\right]}=0,
$$

and looking at its diagonal part. The off-diagonal part of these constraints plays two roles. First, they enforce GL $(D)$ irreducibility on each $R^{I_{1} \ldots I_{n}}$ as a spacetime tensor. They further tell that, for given $n$, all the $\left(\begin{array}{l}s \\ n\end{array}\right)$ seemingly different $R^{I_{1} \ldots I_{n}}$ actually represent the same spacetime tensor with the same Young tableau, and they only differ in the $\psi_{I}$ structure. To see this it would be much easier to go back to the $(D+1)$-dimensional picture, but we can still analyze $(3.12)$ in a bit more detail. For $K \neq L$ the operator $J_{K}^{L}$ removes a spacetime index from column $L$ and antisymmetrizes it within column $K$. Equation (3.12) can be split in three cases:

- $L \in\left\{I_{1} \ldots I_{n}\right\}$ : removing an index from a short column, and placing it in any other column where it is antisymmetrized, gives zero.

- $K, L \notin\left\{I_{1} \ldots I_{n}\right\}$ : removing an index from a long column and antisymmetrizing it within a long column gives zero.

- $L \notin\left\{I_{1} \ldots I_{n}\right\}, K \in\left\{I_{1} \ldots I_{n}\right\}$ : removing an index from a long column $L$ and antisymmetrizing it within a short column $K$ equates it to another $R^{I_{1} \ldots I_{n}}$ tensor having a short $L$ column and long $K$ one.

The first two conditions amount to $\operatorname{GL}(D)$ irreducibility, while the third says that the various $R^{I_{1} \ldots I_{n}}$ at fixed $n$ differ only in naming which columns are the short ones, i.e. they only differ in the $\psi_{I}$ species.

Summarizing the whole content of (3.12) we have that, for given $n$, any $R^{I_{1} \ldots I_{n}}$ is represented by the same spacetime tensor whose GL $(D)$ Young tableau is obtained from a rectangular $d \times s$ one by removing $n$ cells from the bottom row

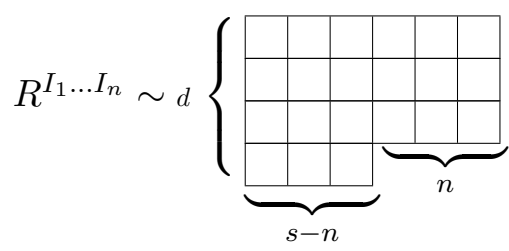

\footnotetext{
${ }^{4}$ We refer to columns, using a Young tableau language, to denote blocks of antisymmetric indices.
} 
The entire field content is then given by the $s+1$ tensors $\left\{R, R^{I}, R^{I J}, \ldots, R^{I_{1} \ldots I_{s}}\right\}$. Starting from the maximal rank one, $R$ with rectangular $d \times s$ Young tableau, one goes down by removing one by one the cells of the bottom row until $R^{I_{1} \ldots I_{s}}$, with rectangular $(d-1) \times s$ diagram, is reached. This final picture is clear having in mind the dimensional reduction of a tensor with a rectangular Young tableau.

Having treated the tensor structure of the states of the physical Hilbert space, the other independent constraints, namely $\mathcal{Q}_{I}$ and $\overline{\mathcal{K}}^{I J}$, give the dynamics for the system. The constraints on $R^{I_{1} \ldots I_{n}}$ read

$$
\begin{aligned}
Q_{K} R^{I_{1} \ldots I_{n}}+m(-)^{s d+n+1} n \delta_{K}^{\left[I_{1}\right.} R^{\left.I_{2} \ldots I_{n}\right]} & =0 \\
\operatorname{tr}^{K L} R^{I_{1} \ldots I_{n}}-R^{K L I_{1} \ldots I_{n}} & =0 .
\end{aligned}
$$

The first equation gives integrability conditions and, in the massive case, relates higher rank tensors to the lower rank ones via successive derivatives, while the second equation enforces trace conditions that contain the truly dynamical equations. The mass parameter in the integrability condition above gives different physical interpretations to the tensors $R^{I_{1} \ldots I_{n}}$, depending whether it vanishes or not. For this reason we shall now treat separately the massive case and its massless limit.

\subsubsection{The massive case: Pauli-Fierz}

When the mass parameter is nonzero, we can invert the first equation in (3.13) to get higher rank curvatures in terms of lower ones

$$
R^{I_{1} \ldots I_{n}}=(-)^{s d+n+1} \frac{1}{m(s-n)} Q_{K} R^{K I_{1} \ldots I_{n}} .
$$

This can be iterated until they are all expressed in terms of the last one $R^{I_{1} \ldots I_{s}}$, that is the only independent field left, giving all the curvatures as

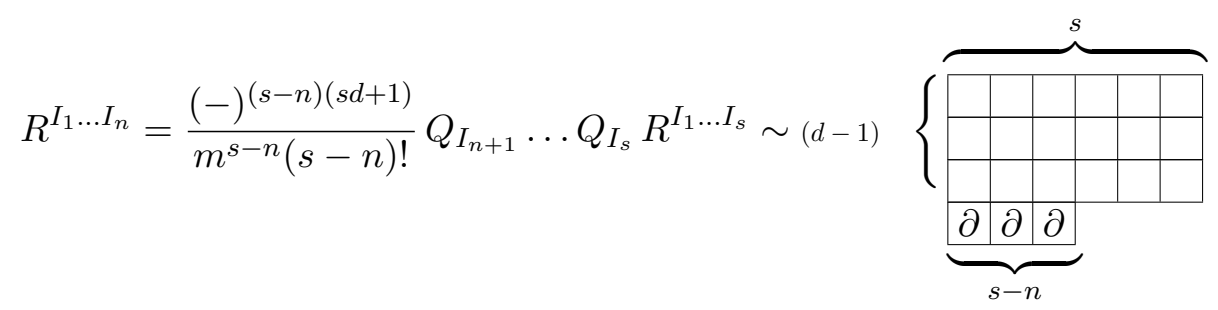

Since every equation can be cast in terms of $R^{I_{1} \ldots I_{s}}$ only, it is convenient to use the $\mathrm{SU}(s)$ invariant symbol $\epsilon^{I_{1} \ldots I_{s}}$ to dualize all the fields as

$$
R^{I_{1} \ldots I_{n}}=\epsilon^{I_{1} \ldots I_{n} J_{1} \ldots J_{s-n}} \widetilde{R}_{J_{1} \ldots J_{s-n}} .
$$

In particular we reserve a different name for the independent field: $\widetilde{R}=\phi$ (the one corresponding to $\left.R^{I_{1} \ldots I_{s}}\right)$. In this dual picture, the Young tableau for $\widetilde{R}_{I_{1} \ldots I_{n}}$ is given by adding $n$ cells in a $d$-th row to the $(d-1) \times s$ box diagram of $\phi$ 


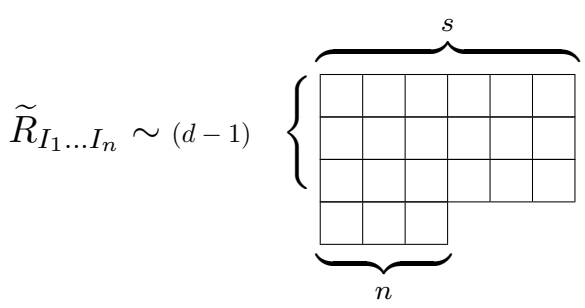

For sake of completeness we give here all the relevant constraint equations for the fields in the dual basis

$$
\begin{aligned}
\left(J_{K}^{L}-d \delta_{K}^{L}\right) \widetilde{R}_{I_{1} \ldots I_{n}}+(n+1) \delta_{[K}^{L} \widetilde{R}_{\left.I_{1} \ldots I_{n}\right]} & =0 \\
Q_{K} \widetilde{R}_{I_{1} \ldots I_{n}}+m(-)^{s d}(n+1) \widetilde{R}_{K I_{1} \ldots I_{n}} & =0 \\
\operatorname{tr}^{K L} \widetilde{R}_{I_{1} \ldots I_{n}}-\delta_{\left[I_{1}\right.}^{K} \delta_{I_{2}}^{L} \widetilde{R}_{\left.I_{3} \ldots I_{n}\right]} & =0
\end{aligned}
$$

The first equation reproduces the field content just described. The $Q$ equations can be solved iteratively to give

$$
\widetilde{R}_{I_{1} \ldots I_{n}}=\frac{(-)^{s d+1}}{m n} Q_{\left[I_{1}\right.} \widetilde{R}_{\left.I_{2} \ldots I_{n}\right]}=\cdots=\frac{(-)^{n(s d+1)}}{m^{n} n !} Q_{I_{1}} \ldots Q_{I_{n}} \phi
$$

and the consistency condition $Q_{K} \widetilde{R}_{I_{1} \ldots I_{n}}-Q_{[K} \widetilde{R}_{\left.I_{1} \ldots I_{n}\right]}=0$ is trivially satisfied due to the anticommuting nature of the $Q_{I}$ 's. ${ }^{5}$

At this point the only fields needed are $\phi$ and the curvatures

$$
\widetilde{R}_{I}=\frac{(-)^{s d+1}}{m} Q_{I} \phi, \quad \widetilde{R}_{I J}=\frac{1}{2 m^{2}} Q_{I} Q_{J} \phi .
$$

The relevant field equations come from the trace constraints in (3.17) for $n=0,1,2$ while all the higher order constraints will be derivatives of the field equations themselves. Explicitly, the relevant trace constraints are

$$
\begin{array}{r}
\operatorname{tr}^{K L} \phi=0, \quad \operatorname{tr}^{K L} \widetilde{R}_{I}=0 \\
\operatorname{tr}^{K L} \widetilde{R}_{I J}-\delta_{[I}^{K} \delta_{J]}^{L} \phi=0 .
\end{array}
$$

The first equation tells that the field $\phi$ is completely traceless, while the second one reads $\operatorname{tr}^{K L} Q_{I} \phi=0$. Using the [tr, $Q$ ] algebra in (3.9) and $\operatorname{tr}^{I J} \phi=0$ one finds $\bar{Q}^{I} \phi=0$, i.e. $\phi$ is divergence-free. At this point the last equation in (3.20) becomes trivial for $\{K L\} \notin\{I J\}$. The only nontrivial part sits in its contraction

$$
\operatorname{tr}^{I J} \widetilde{R}_{I J}-\frac{s(s-1)}{2} \phi=0 .
$$

By using the $[\operatorname{tr}, Q]$ algebra as above and $\left\{Q_{I}, \bar{Q}^{J}\right\}=2 \delta_{I}^{J} H$, as long as the previous trace and divergence constraints are imposed, it simply becomes the massive Klein-Gordon equation

$$
\left(p^{2}+m^{2}\right) \phi=0 .
$$

\footnotetext{
${ }^{5}$ Note that on $(\mathrm{A}) \mathrm{dS}$ spaces the $Q_{I}$ 's do not anticommute, and this will become a nontrivial consistency condition of the solution.
} 
We have thus shown that the physical content of the model reduces to a single field $\phi$ described by a rectangular $(d-1) \times s$ Young tableau

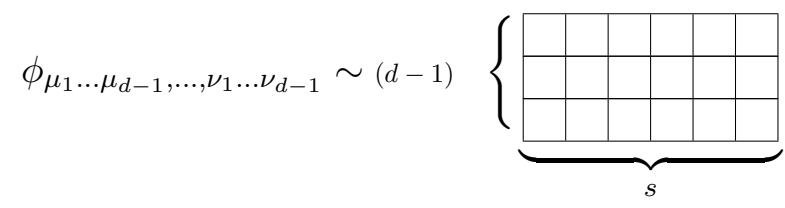

obeying the Fierz-Pauli massive equations [34], that in our language read

$$
\operatorname{tr}^{I J} \phi=0, \quad \bar{Q}^{I} \phi=0, \quad\left(p^{2}+m^{2}\right) \phi=0 .
$$

In the more explicit tensorial notation they take the form

$$
\begin{aligned}
\phi^{\mu}{ }_{\mu_{2} \ldots \mu_{d-1}, \ldots, \mu \nu_{2} \ldots \nu_{d-1}} & =0 \\
\partial^{\mu} \phi_{\mu \mu_{2} \ldots \mu_{d-1}, \ldots, \nu_{1} \ldots \nu_{d-1}} & =0 \\
\left(-\square+m^{2}\right) \phi_{\mu_{1} \ldots \mu_{d-1}, \ldots, \nu_{1} \ldots \nu_{d-1}} & =0 .
\end{aligned}
$$

Having analyzed the massive case, we can now turn to the somehow richer massless limit.

\subsubsection{The massless limit: Fronsdal-Labastida multiplets}

If we set the mass parameter to zero, the only constraint equations that change are the $Q$ ones. Since, as we will see, in this case one has $s+1$ different physical fields, there is no real advantage in using the dual basis, and we return to the original one, i.e. $R^{I_{1} \ldots I_{n}}$. The irreducibility constraints $\mathcal{J}_{I}^{J}$ are exactly the same and as before they yield

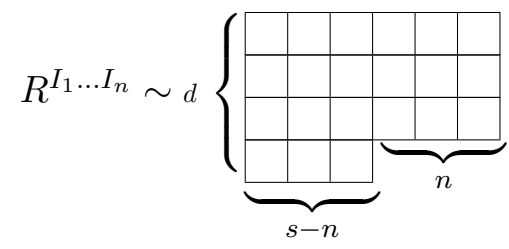

The remaining equations now read

$$
\begin{aligned}
Q_{K} R^{I_{1} \ldots I_{n}} & =0, \\
\operatorname{tr}^{K L} R^{I_{1} \ldots I_{n}}-R^{K L I_{1} \ldots I_{n}} & =0 .
\end{aligned}
$$

The $Q$ equations now tell us that each curvature separately obeys Bianchi integrability conditions, and indeed we shall integrate them in terms of $s+1$ different massless potentials. Part of the analysis is now strictly analogous to what we reviewed in section 2: we introduce two higher derivative operators

$$
q=\frac{1}{s !} \epsilon^{I_{1} \ldots I_{s}} Q_{I_{1}} \ldots Q_{I_{s}}, \quad q^{I J}=\frac{1}{(s-2) !} \epsilon^{I J I_{3} \ldots I_{s}} Q_{I_{3}} \ldots Q_{I_{s}}
$$

and we use $q$ to solve the integrability constraints as

$$
R^{I_{1} \ldots I_{n}}=q \varphi^{I_{1} \ldots I_{n}} .
$$


To understand the tensor structure of $\varphi^{I_{1} \ldots I_{n}}$, notice that $\left[J_{I}^{J}, q\right]=\delta_{I}^{J} q$. This means that at the level of Young tableaux $q$ attaches $s$ cells at the bottom of the $s$ columns of the diagram. Hence, the $\varphi^{I_{1} \ldots I_{n}}$ Young tableau can be obtained from the tableau of $R^{I_{1} \ldots I_{n}}$ by stripping off one cell from the bottom of each column. In general the resulting structure will be

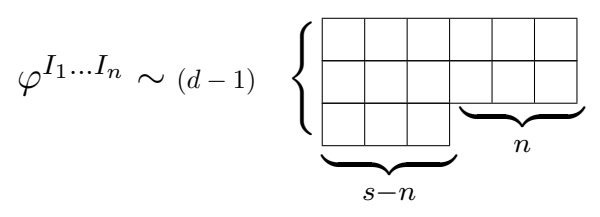

and the pictorial relation between curvature and potential is as follows

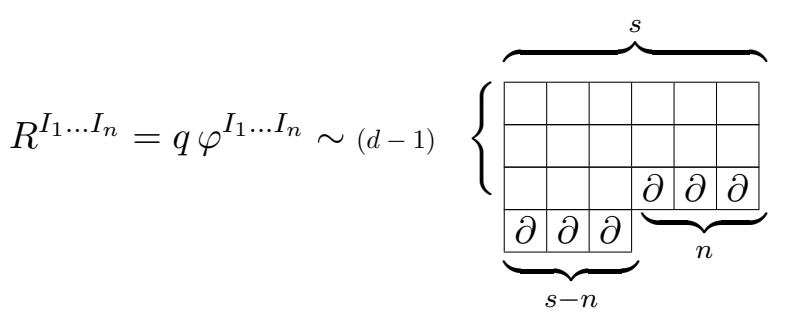

Something slightly different happens in $D=3$, that is $d=2$ : the $(d-2) \times s$ box diagram is now empty, and one has symmetric tensors of spin ranging from zero to $s$, namely

$$
\varphi^{I_{1} \ldots I_{n}} \sim \underbrace{\square \mid \square}_{s-n}, \quad D=3 .
$$

Once the curvatures are written in terms of potentials, the field equations take the form

$$
\operatorname{tr}^{K L} q \varphi^{I_{1} \ldots I_{n}}-q \varphi^{K L I_{1} \ldots I_{n}}=0
$$

and they are higher derivative equations. Following the derivation sketched in section 2, we notice that

$$
\begin{aligned}
\operatorname{tr}^{K L} q & =q^{K L} G, \quad G=-2 H+Q_{I} \bar{Q}^{I}+\frac{1}{2} Q_{I} Q_{J} \operatorname{tr}^{I J} \\
q \varphi^{K L I_{1} \ldots I_{n}} & =\frac{1}{2} q^{K L} Q_{I} Q_{J} \varphi^{I J I_{1} \ldots I_{n}}
\end{aligned}
$$

where $G$ is the Fronsdal-Labastida operator. The equations (3.28) can then be recast as

$$
q^{K L}\left(G \varphi^{I_{1} \ldots I_{n}}-\frac{1}{2} Q_{I} Q_{J} \varphi^{I J I_{1} \ldots I_{n}}\right)=0
$$

The expression inside the bracket mixes different potentials, but it is possible to decouple them recursively with a field redefinition:

$$
\varphi^{I_{1} \ldots I_{n}}=\tilde{\varphi}^{I_{1} \ldots I_{n}}+\sum_{j=1}^{m} \alpha_{j}^{(n)} g_{K_{1} L_{1}} \ldots g_{K_{j} L_{j}} \tilde{\varphi}^{K_{1} L_{1} \ldots K_{j} L_{j} I_{1} \ldots I_{n}} .
$$


Namely, one starts from $\varphi^{I_{1} \ldots I_{s}}=\tilde{\varphi}^{I_{1} \ldots I_{s}}$ and $\varphi^{I_{1} \ldots I_{s-1}}=\tilde{\varphi}^{I_{1} \ldots I_{s-1}}$ and goes down until $\varphi^{I_{1} \ldots I_{n}}$ with $n=s-2 m$ or $n=s-1-2 m$ in (3.31). The $\alpha_{j}^{(n)}$ coefficients can be found by recursion and read

$$
\alpha_{0}^{(n)}:=1, \quad \alpha_{j}^{(n)}=\frac{\alpha_{j-1}^{(n+2)}}{4 j\left(j+n-s+\frac{1}{2}\right)},
$$

so that

$$
\alpha_{j}^{(n)}=\frac{1}{4^{j} j ! \prod_{l=1}^{j}\left(2 j-l+n-s+\frac{1}{2}\right)} .
$$

Once we have $s+1$ decoupled equations

$$
q^{K L} G \tilde{\varphi}^{I_{1} \ldots I_{n}}=0
$$

we can drop the tildes and, since $q^{I J} \sim Q^{s-2}$, we can locally parametrize its kernel as $Q^{3} \rho$, namely

$$
G \varphi^{I_{1} \ldots I_{n}}=Q_{I} Q_{J} Q_{K} \rho^{I J K \mid I_{1} \ldots I_{n}},
$$

that are nothing but Fronsdal-Labastida equations for the mixed symmetry tensors $\varphi^{I_{1} \ldots I_{n}}$ with compensators. We can see from (3.34) that for each $\varphi^{I_{1} \ldots I_{n}}$ there are different compensator structures: indeed their Young tableaux are obtained from the corresponding $\varphi$ diagrams by removing three cells in the $I J K$ columns. Since there is no symmetry relation between the two sets $I J K$ and $I_{1} \ldots I_{n}$, one has different tensors for $\rho$ whether some $I J K$ coincide with some $I_{k}$ or not. In the next subsection we will provide some explicit examples of the various structures that can arise.

\subsubsection{Gauge invariance}

As expected in a theory of massless fields, the equations giving the curvatures in terms of the potentials admit a gauge symmetry that leaves the curvatures invariant. Since $q Q_{I}=Q_{I} q=0$ it is easy to see that $\delta R^{I_{1} \ldots I_{n}}=0$ if we vary the gauge field as

$$
\delta \varphi^{I_{1} \ldots I_{n}}=Q_{K} \Lambda^{K \mid I_{1} \ldots I_{n}} .
$$

As it was the case for the compensators, the Young diagram of the gauge parameter is obtained from the gauge field one by removing one cell in all possible ways. This produces different gauge parameters whether the index $K$ coincides or not with one of the $I_{k}$. The curvatures are then gauge invariant under (3.35), but $G \varphi^{I_{1} \ldots I_{n}}$ is not. Nonetheless the compensated equations are gauge invariant if we give the compensators the following transformation rule

$$
\delta \rho^{I J K \mid I_{1} \ldots I_{n}}=\frac{1}{2} \operatorname{tr}^{[I J} \Lambda^{K] \mid I_{1} \ldots I_{n}} .
$$

One can then partially gauge fix the theory setting the compensators to zero to recover the usual Fronsdal-Labastida equations

$$
G \varphi^{I_{1} \ldots I_{n}}=0
$$

that are gauge invariant for traceless gauge parameters: $\operatorname{tr}^{[I J} \Lambda^{K] \mid I_{1} \ldots I_{n}}=0$. 
Now we would like to give some explicit examples to clarify which tensor structures appear for various spins and dimensions. If we take for instance $s=4$ in $D=5$, i.e. $d=3$ we have in the massive case the single field

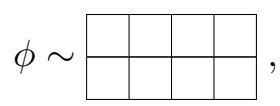

while in the massless limit one obtains the following multiplet of massless FronsdalLabastida gauge fields

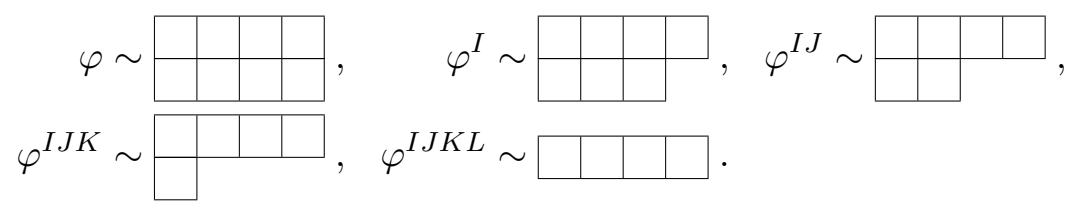

Taking for instance $\varphi^{I J}$, its gauge invariance is parametrized by two different set of parameters $\Lambda^{K \mid I J}$

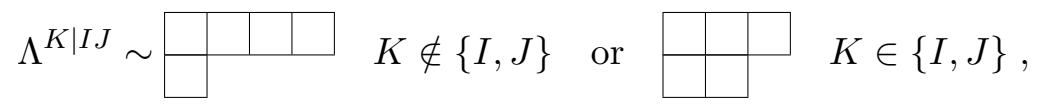

while the compensators appearing in (3.34) can be

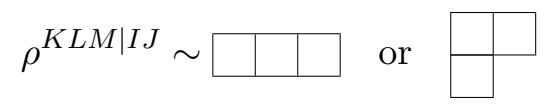

whether one or two indices in $K L M$ coincide with $I J$.

\section{Coupling to curved space: (A)dS manifolds}

In the previous section we have described a massive higher spinning particle in flat, odd spacetime dimensions, together with its massless limit, by dimensionally reducing a massless model defined in a flat even-dimensional space. It is known that the latter can be coupled to (A)dS spaces, and more generally to conformally flat spaces. Thus, it is natural to investigate possible extensions of our model to curved spaces. We focus in particular to (A)dS backgrounds, which are the ones that appear in the construction of the Vasiliev's interacting models.

We proceed as follows. We covariantize the constraints that define our model. Then, considering a curved metric, we check if the algebra remains first class. If that happens to be true, it means that the gauge symmetries defining the model are not broken by the curvature, and the model is viable. Indeed we find that a coupling to (A)dS is allowed.

In order to deform the quantum constraint algebra to include a $D$ dimensional curved target space, we start from the $\mathrm{SO}(N)$ generators, where the task is easy. In this case we only need to use worldline fermions with flat indices. For this purpose we use the first part of the greek alphabet to indicate flat indices, i.e. $\psi_{i}^{\alpha}$ with $\alpha=0,1, \ldots, D-1$, (curved indices can then be obtained by using a vielbein $e_{\mu}{ }^{\alpha}$ and its inverse $e_{\alpha}{ }^{\mu}$ ). We define the quantum $\mathrm{SO}(N)$ generators by

$$
\mathcal{J}_{i j}=\frac{i}{2}\left[\psi_{i}^{\alpha}, \psi_{j \alpha}\right]+\frac{i}{2}\left[\theta_{i}, \theta_{j}\right]:=J_{i j}+L_{i j}
$$


which are seen to satisfy the $\mathrm{SO}(N)$ algebra (indeed there is no change with respect to the calculation in flat space). The ordering prescription used in (4.1) is uniquely fixed by the $\mathrm{SO}(N)$ algebra. Then, we covariantize the susy generators by replacing the linear momentum $p_{\mu}$ with the covariant momentum $\pi_{\mu}$, i.e.

$$
\begin{aligned}
\mathcal{Q}_{i} & =\psi_{i}^{\alpha} e_{\alpha}{ }^{\mu} \pi_{\mu}+\theta_{i} m:=Q_{i}+\theta_{i} m \\
\pi_{\mu} & =p_{\mu}-\frac{1}{2} \omega_{\mu \alpha \beta} M^{\alpha \beta}
\end{aligned}
$$

where $M^{\alpha \beta}=\frac{i}{2}\left[\psi_{i}^{\alpha}, \psi_{i}^{\beta}\right]$ are the Lorentz generators in the multispinor representation and $\omega_{\mu \alpha \beta}$ the spin connection. Since by definition the $Q_{i}$ 's do not involve $\theta$ 's, they are nothing but the massless susy constraints appearing in ref. [17]. They satisfy the commutation rule

$$
\left\{Q_{i}, Q_{j}\right\}=2 \delta_{i j} H_{0}+\frac{i}{2} \psi_{i}^{\alpha} \psi_{j}^{\alpha^{\prime}} R_{\alpha \alpha^{\prime} \beta \beta^{\prime}} M^{\beta \beta^{\prime}}
$$

where $H_{0}=\frac{1}{2}\left(\pi^{\alpha} \pi_{\alpha}-i \omega^{\beta}{ }_{\beta \alpha} \pi^{\alpha}\right)$ and $R_{\alpha \beta \gamma \delta}$ is the Riemann curvature tensor. For the full susy constraints we thus have

$$
\left\{\mathcal{Q}_{i}, \mathcal{Q}_{j}\right\}=2 \delta_{i j}\left(H_{0}+\frac{1}{2} m^{2}\right)+\frac{i}{2} \psi_{i}^{\alpha} \psi_{j}^{\alpha^{\prime}} R_{\alpha \alpha^{\prime} \beta \beta^{\prime}} M^{\beta \beta^{\prime}}
$$

which imply that $\mathcal{Q}_{i}, \mathcal{J}_{i j}$ and a suitably chosen hamiltonian constraint $\mathcal{H}$ cannot possibly form an algebra of first class constraints for a generic background. However restricting to maximally symmetric spaces

$$
R_{\alpha \beta \gamma \delta}=b\left(\eta_{\alpha \gamma} \eta_{\beta \delta}-\eta_{\alpha \delta} \eta_{\beta \gamma}\right)
$$

one finds

$$
\left\{\mathcal{Q}_{i}, \mathcal{Q}_{j}\right\}=2 \delta_{i j}\left(\tilde{H}+\frac{1}{2} m^{2}\right)+\frac{b}{2}\left(\delta_{i j} J_{k k^{\prime}} J_{k k^{\prime}}-J_{i k} J_{j k}-J_{j k} J_{i k}\right)
$$

where

$$
\tilde{H}=H_{0}-\frac{b}{4} J_{k k^{\prime}} J_{k k^{\prime}}-b A(D)
$$

The second term is an improvement term that is added and subtracted in (4.7) to achieve $\left[\tilde{H}, Q_{i}\right]=\left[\tilde{H}, J_{i j}\right]=0$, and $A(D)=(2-N) \frac{D}{8}-\frac{D^{2}}{8}$ is a quantum effect due to operatorial ordering. The essential point to observe is that the right hand side of eq. (4.7) is expressed with respect to the $J_{i j}$ operators that are not the constraints operators $\mathcal{J}_{i j}=J_{i j}+L_{i j}$ that we need to impose to get the massive HS equations of motion. So we ought to rewrite such relations in terms of the $\mathcal{J}_{i j}$ operators. Using the commutator rule $\left\{\theta_{i}, \theta_{j}\right\}=\delta_{i j}$, we find

$$
L_{i k} L_{j k}+L_{j k} L_{i k}=\frac{N-1}{2} \delta_{i j}, \quad L_{k k^{\prime}} L_{k k^{\prime}}=\frac{N(N-1)}{4}
$$

and

$$
\begin{aligned}
J_{i k} J_{j k}+J_{j k} J_{i k} & =\mathcal{J}_{i k} \mathcal{J}_{j k}+\mathcal{J}_{j k} \mathcal{J}_{i k}-2 L_{i k} \mathcal{J}_{j k}-2 L_{j k} \mathcal{J}_{i k}+\frac{N-1}{2} \delta_{i j} \\
J_{k k^{\prime}} J_{k k^{\prime}} & =\mathcal{J}_{k k^{\prime}} \mathcal{J}_{k k^{\prime}}-2 L_{k k^{\prime}} \mathcal{J}_{k k^{\prime}}+\frac{N(N-1)}{4}
\end{aligned}
$$


Thus we can rewrite (4.7) as

$$
\begin{aligned}
\left\{\mathcal{Q}_{i}, \mathcal{Q}_{j}\right\}= & 2 \delta_{i j} \mathcal{H} \\
& +\frac{b}{2}\left[\delta_{i j}\left(\mathcal{J}_{k k^{\prime}}-2 L_{k k^{\prime}}\right) \mathcal{J}_{k k^{\prime}}-\mathcal{J}_{i k} \mathcal{J}_{j k}-\mathcal{J}_{j k} \mathcal{J}_{i k}+2 L_{i k} \mathcal{J}_{j k}+2 L_{j k} \mathcal{J}_{i k}\right]
\end{aligned}
$$

and recover a first class system with the hamiltonian constraint $\mathcal{H}$ defined by

$$
\begin{aligned}
\mathcal{H} & =H_{0}+\frac{1}{2} m^{2}-\frac{b}{4}\left(\mathcal{J}_{k k^{\prime}}-2 L_{k k^{\prime}}\right) \mathcal{J}_{k k^{\prime}}-b\left(A(D)+\frac{N-1}{8}\right) \\
& =\tilde{H}+\frac{1}{2} m^{2}+\frac{b}{16}(N-1)(N-2) .
\end{aligned}
$$

Indeed, since $\mathcal{H}$ and $\tilde{H}$ only differ by a constant term, we have

$$
\left[\mathcal{H}, \mathcal{Q}_{i}\right]=\left[\mathcal{H}, \mathcal{J}_{i j}\right]=0
$$

and $\mathcal{H}$ is a central element of the algebra (in fact, $\mathcal{H}$ separately commutes with $\left.Q_{i}, \theta_{i}, J_{i j}, L_{i j}\right)$.

To summarize, we have seen that the constraints $\mathcal{J}_{i j}, \mathcal{Q}_{i}$, and $\mathcal{H}$ identified above form a first class system with nontrivial structure functions. The latter arise because of the curvature of the (A)dS spaces, encoded in the parameter $b$ that is related to the scalar curvature by $R=D(D-1) b$. We conclude that the massive higher spinning particle can be defined on (A)dS spaces.

A classical action for the model can be written down immediately using the classical limit of the above constraints, and the corresponding lagrangian read as

$$
\begin{aligned}
L= & p_{\mu} \dot{x}^{\mu}+\frac{i}{2} \psi_{i \alpha} \dot{\psi}_{i}^{\alpha}+\frac{i}{2} \theta_{i} \dot{\theta}_{i}-e \underbrace{\frac{1}{2}\left(\pi_{\mu} \pi^{\mu}-\frac{b}{2} J_{i j} J_{i j}+m^{2}\right)}_{\mathcal{H}^{c l}} \\
& -i \chi_{i} \underbrace{\left(e_{\alpha}{ }^{\mu} \pi_{\mu} \psi_{i}^{\alpha}+m \theta_{i}\right)}_{\mathcal{Q}_{i}^{c l}}-\frac{1}{2} a_{i j} \underbrace{\left(i \psi_{i}^{\alpha} \psi_{j \alpha}+i \theta_{i} \theta_{j}\right)}_{\mathcal{J}_{i j}^{c l}} .
\end{aligned}
$$

\subsection{Light cone analysis}

The lagrangian (4.12) describes a massive HS particle in odd dimensional (A)dS spaces. As stressed, the constraint algebra is first class, so that its gauging is consistent and the model is viable. To check that the HS particle indeed carries nontrivial degrees of freedom, we perform a light cone analysis at the classical level. It goes in a way similar to the one presented earlier for flat space, and we highlight just the main points.

To proceed we follow [35]. For simplicity we set $b=-1$ in (4.6) and use the Poincarè parametrization of the AdS space with $d s^{2}=\left(-\left(d x^{0}\right)^{2}+\left(d x^{1}\right)^{2}+\ldots+\left(d x^{D-3}\right)^{2}+d z^{2}+\right.$ $\left.\left(d x^{D-1}\right)^{2}\right) / z^{2}$, where a special role is played by the coordinate $x^{D-2}:=z$. As before we set $x^{ \pm}=\left(x^{D-1} \pm x^{0}\right) / \sqrt{2}$, so that $d s^{2}=\left(2 d x^{+} d x^{-}+d x^{A} d x^{A}+d z^{2}\right) / z^{2}$ with $A=1,2, \ldots D-3$, and consider $x^{+}$as the light cone time. We also use an index $a=(A, D-2)$ that runs over $D-2$ values to include the one corresponding to the coordinate $x^{D-2}:=z$, so that one could write the metric in the form $d s^{2}=\left(2 d x^{+} d x^{-}+d x^{a} d x^{a}\right) / z^{2}$ as well. 
Now we make the gauge choice $x^{+}=\tau$. Correspondingly one may solve the hamiltonian constraint $\mathcal{H}^{c l}$ by $p_{+}=-\frac{1}{2 z^{2} \pi_{-}}\left(z^{2} \pi_{a} \pi_{a}+\frac{1}{2} J_{i j}^{2}+m^{2}\right)+\frac{1}{2} \omega_{+\alpha \beta} M^{\alpha \beta}$. In a similar way we set $\psi_{i}^{+}=0$ by a gauge choice, and solve $\mathcal{Q}_{i}^{c l}$ by $\psi_{i}^{-}=-\frac{1}{e_{-}{ }^{\mu} \pi_{\mu}}\left(e_{a}{ }^{\mu} \pi_{\mu} \psi_{i}^{a}+m \theta_{i}\right)$. This leaves a lagrangian of the form ${ }^{6}$

$$
L=p_{-} \dot{x}^{-}+p_{a} \dot{x}^{a}+\frac{i}{2} \psi_{i a} \dot{\psi}_{i}^{a}+\frac{i}{2} \theta_{i} \dot{\theta}_{i}+p_{+}-\frac{1}{2} a_{i j} \underbrace{\left(i \psi_{i}^{a} \psi_{j a}+i \theta_{i} \theta_{j}\right)}_{\mathcal{J}_{i j}}
$$

where the remaining algebraic constraints related to the $\mathrm{SO}(N)$ charges are exactly the same as the one present in the flat space discussion. Their treatment proceeds in the same way, so that we conclude that the number of degrees of freedom remains unchanged when passing from flat space to an AdS background.

\subsection{Covariant analysis: massive case}

We have shown that the deformed constraint superalgebra remains first class on (A)dS backgrounds. This ensures that, in the complex U(s) basis, the independent constraints $\mathcal{J}_{I}^{J}, \overline{\mathcal{K}}^{I J}, \mathcal{Q}_{I}$ produce consistent and covariant dynamical equations. In order to find them we split again the operators according to the $\theta$ content as done in section 3.2. The field content in (A)dS remains unchanged, at the level of $R^{I_{1} \ldots I_{n}}$ tensors, since the $\mathrm{SO}(N)$ generators and the corresponding constraint analysis are unmodified. The dynamics is governed as in the flat case by the integrability and trace conditions as in (3.13), the difference with respect to flat space being the covariant momenta inside the $Q_{I}$, that give non trivial anticommutators. Indeed, the algebra of $D$-dimensional operators reported in (3.8) and (3.9) remains unchanged in (A)dS, while (3.10) becomes

$$
\begin{aligned}
& \left\{Q_{I}, Q_{J}\right\}=b\left(g_{I K} J_{J}^{K}+g_{J K} J_{I}^{K}\right), \quad\left\{\bar{Q}^{I}, \bar{Q}^{J}\right\}=-b\left(\operatorname{tr}^{I K} J_{K}^{J}+\operatorname{tr}^{J K} J_{K}^{I}\right), \\
& \left\{Q_{I}, \bar{Q}^{J}\right\}=2 \delta_{I}^{J} H_{0}-\frac{b}{2}\left(J_{I}^{K} J_{K}^{J}+J_{K}^{J} J_{I}^{K}-2 g_{I K} \operatorname{tr}^{J K}+(4-4 d-s) J_{I}^{J}-\delta_{I}^{J} J_{K}^{K}\right),
\end{aligned}
$$

where we prefer to give the last relation in terms of the minimal $H_{0}$, since it is the operator represented by the minimal covariant laplacian $2 H_{0}=-\nabla^{2}$. In the massive case the analysis proceeds along the same steps described in section 3.2. The only independent field is $\widetilde{R}=\phi$, with rectangular $(d-1) \times s$ Young tableau, obeying

$$
\begin{gathered}
\operatorname{tr}^{K L} \phi=0, \quad \frac{1}{m} \operatorname{tr}^{K L} Q_{I} \phi=0, \\
\frac{1}{2 m^{2}} \operatorname{tr}^{K L} Q_{I} Q_{J} \phi-\delta_{[I}^{K} \delta_{J]}^{L} \phi=0 .
\end{gathered}
$$

The remaining trace conditions are automatically satisfied, provided that the three above equations hold. This fact, along with the mutual consistency of the $Q_{I}$ integrability constraints, is highly nontrivial on (A)dS. The light cone analysis of the previous subsection guarantees that the propagating degrees of freedom are conserved with respect to the flat case, ensuring that the model is not empty in (A)dS.

\footnotetext{
${ }^{6}$ On fermions the indices are to be considered as flat.
} 
The first equation tells us as before that $\phi$ is traceless ${ }^{7}$ and, since the $[\operatorname{tr}, Q]$ algebra is unchanged, the second one turns again into a divergence constraint

$$
\bar{Q}^{I} \phi=0 \quad \leftrightarrow \quad \nabla^{\alpha} \phi_{\alpha \alpha_{2} \ldots \alpha_{d-1}, \ldots, \beta_{1} \ldots \beta_{d-1}}=0
$$

The main difference with respect to the flat case appears in the third equation, due to the deformed $\left\{Q_{I}, \bar{Q}^{J}\right\}$ algebra, as can be seen from (4.14). In order to get the Klein-Gordon equation from (4.15), let us manipulate the $\operatorname{tr} Q Q$ term. Pushing the trace operator through the supercharges, and using $\operatorname{tr}^{I J} \phi=\bar{Q}^{I} \phi=0$ we get

$$
\operatorname{tr}^{K L} Q_{I} Q_{J} \phi=2 \delta_{I}^{[L}\left\{\bar{Q}^{K]}, Q_{J}\right\} \phi .
$$

We use now the superalgebra (4.14), along with the $\mathrm{U}(s)$ constraint $J_{I}^{J} \phi=(d-1) \delta_{I}^{J} \phi$, to obtain

$$
2 \delta_{I}^{[L}\left\{\bar{Q}^{K]}, Q_{J}\right\} \phi=2 \delta_{I}^{[L} \delta_{J}^{K]}\left(2 H_{0}+b(d-1)(d-1+s)\right) \phi .
$$

Finally, inserting the above result in (4.15) yields

$$
\left(-2 H_{0}-b(d-1)(d-1+s)-m^{2}\right) \phi=0,
$$

i.e. the covariant Klein-Gordon equation with the mass term shifted by a geometric contribution, thus completing the triplet of massive Fierz-Pauli conditions in (A)dS:

$$
\begin{aligned}
\phi^{\beta}{ }_{\alpha_{2} \ldots \alpha_{d-1}, \ldots, \beta \beta_{2} \ldots \beta_{d-1}} & =0 \\
\nabla^{\alpha} \phi_{\alpha \alpha_{2} \ldots \alpha_{d-1}, \ldots, \beta_{1} \ldots \beta_{d-1}} & =0 \\
\left(\nabla^{2}-b(d-1)(d-1+s)-m^{2}\right) \phi_{\alpha_{1} \ldots \alpha_{d-1}, \ldots, \beta_{1} \ldots \beta_{d-1}} & =0 .
\end{aligned}
$$

We are now ready to analyze the massless limit, that is considerably more involved. In general we will not find Fronsdal-Labastida equations for mixed symmetry gauge fields, and we will limit ourselves to work out an explicit example.

\subsection{Massless limit: an example}

We give an interesting and non trivial example of what the model describes in the "massless" limit $m=0$. In such a limit the dynamical equations in (A)dS reduce to

$$
\begin{aligned}
Q_{K} R^{I_{1} \ldots I_{n}} & =0, \\
\operatorname{tr}^{K L} R^{I_{1} \ldots I_{n}}-R^{K L I_{1} \ldots I_{n}} & =0 .
\end{aligned}
$$

This form is the same as the flat case one, but now the integrability conditions $Q R=0$ become non trivial, because of the non-vanishing $\{Q, Q\}$ anti-commutator in (4.14), that prevents the operator $q_{0}=\frac{1}{s !} \epsilon^{I_{1} \ldots I_{s}} Q_{I_{1}} \ldots Q_{I_{s}}$ to be annihilated by $Q_{K}$. In the massless even-dimensional (A)dS models of [17] the integrability conditions on HS curvature, described by rectangular GL $(D)$ Young tableaux, were solved. However, unlike [17], in the

\footnotetext{
${ }^{7}$ In this section on (A)dS backgrounds, we treat tensor fields with flat Lorentz indices. Every covariant equation can be rewritten using curved base indices by sending $\eta_{\alpha \beta}$ to $g_{\mu \nu}$.
} 
present work the (A)dS deformation seems to introduce complications for solving the integrability condition of some specific curvature tensors, those described by "pistol-shaped" Young tableaux. This is presumably related to the fact that in AdS the pistol-shaped HS potentials have less gauge symmetries than the corresponding flat space ones ${ }^{8}$ [36-38]. Nonetheless, a light cone analysis applied to the pistol-shaped HS curvature equations, indicates that the corresponding multiplet carries the same number of degrees of freedom in all maximally-symmetric spaces; we will further comment on this point later on.

In the following we discuss the spin-two case in $\mathrm{AdS}_{3}$, where again we refer to the spin as the number of columns in the Young tableaux representing the higher spin curvature(s). Solving the algebraic $\mathrm{U}(2)$ constraints $J_{I}{ }^{J}$, the field content of the model is given by the following curvatures

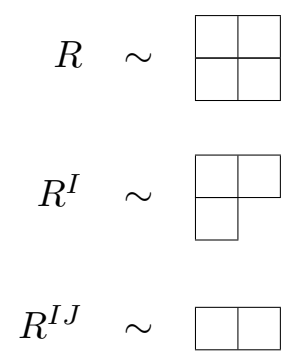

We start analyzing the differential constraints on the first and last curvature since we expect the equations of motion to mix them as in the flat case (being rectangularly shaped we expect no particular difficulties to arise). The differential Bianchi identities $Q_{K} R=$ $0=Q_{K} R^{I J}$ can indeed be solved by introducing a symmetric rank-two gauge potential $\varphi$ and a scalar $\varphi^{I J}$ as follows

$$
R=q_{(2)} \varphi \quad R^{K L}=q_{(2)} \varphi^{K L}
$$

with

$$
q_{(2)}=\frac{1}{2} \epsilon^{I J}\left(Q_{I} Q_{J}-b g_{I J}\right) .
$$

We now use the algebra presented in the previous section to push the trace operator tr through $q_{(2)}$. Firstly let us introduce the AdS generalization of the Fronsdal-Labastida operator $G_{(2)}^{\text {AdS }}$ defined by

$$
\begin{aligned}
& G_{(2)}^{\mathrm{AdS}}:=\frac{1}{2} \epsilon_{K L} \operatorname{tr}^{K L} q_{(2)} \\
& =\left(-2 H_{0}+Q_{I} \bar{Q}^{I}+\frac{1}{2} Q_{I} Q_{J} \operatorname{tr}^{I J}-b g_{I J} \operatorname{tr}^{I J}+\frac{b}{2} J_{K}^{L} J_{L}^{K}-b(d+1) J_{K}^{K}+b(2 d-1)\right)
\end{aligned}
$$

The equations of motion for the above potentials thus are the trace constraints of (4.19). For the scalar potential $\varphi^{I J}:=\epsilon^{I J} \phi$ we simply get

$$
\operatorname{tr}^{I J} q_{(2)} \varphi^{K L}=\epsilon^{I J} G_{(2)}^{\mathrm{AdS}} \varphi^{K L}=0 \quad \Rightarrow \quad\left(\nabla^{2}-3 b\right) \phi=0
$$

\footnotetext{
${ }^{8}$ We thank Per Sundell for this observation.
} 
where on the right hand side we explicitly evaluated the action of the $\mathrm{SO}(4)$ constraints on the scalar potential. Therefore the equations of motions for $R^{I J}$ leave one propagating degree of freedom (DoF), that of a scalar field.

For the rank-two tensor, $\varphi=\frac{1}{2} \phi_{\mu \nu} \epsilon^{I J} \psi_{I}^{\mu} \psi_{J}^{\nu}$, the trace condition amounts to

$$
\operatorname{tr}^{K L} q_{(2)} \varphi=q_{(2)} \varphi^{K L}
$$

and, similarly to the flat space counterpart, we aim to combine $\varphi$ and $\varphi^{K L}$ in a single field. To achieve this task we introduce

$$
\widetilde{\varphi}:=\varphi-\frac{1}{2} g_{I J} \varphi^{I J}
$$

and use the operatorial relation

$$
\left[G_{(2)}^{\mathrm{AdS}}, g\right]=\epsilon^{I J} Q_{I} Q_{J}\left(3-2 d+J_{L}^{L}\right)+(2 d-3) g-b g J_{L}^{L}
$$

with $g=\epsilon^{I J} g_{I J}$ to make $G_{(2)}^{\text {AdS }}$ act on $\varphi^{I J}$, so that, when we put $\varphi^{I J}$ on shell, equation (4.24) reduces to

$$
G_{(2)}^{\operatorname{AdS}} \widetilde{\varphi}=0
$$

which is the Fronsdal equation in (A)dS for a massless spin-two potential, i.e.

$$
\nabla^{2} \phi_{\mu \nu}-\nabla_{\mu} \nabla^{\rho} \phi_{\rho \nu}-\nabla_{\nu} \nabla^{\rho} \phi_{\rho \mu}+\nabla_{\mu} \nabla_{\nu} \phi_{\rho}^{\rho}+2 b\left(g_{\mu \nu} \phi_{\rho}^{\rho}-\phi_{\mu \nu}\right)=0
$$

that in three dimensions carries no DoF's (we dropped the tildes for simplicity).

Finally we now try to integrate $R^{I}$ and impose the trace constraint to extract the last equation of motion. After considering a natural class of ansatze, we could not find a nontrivial solution to $Q_{K} R^{I}=0$, and it seems that $R^{I}$ cannot be integrated in terms of a potential. Nonetheless, a direct light cone check shows that the constraints $Q_{J} R^{I}=$ $\operatorname{tr}^{J K} R^{I}=0$ leave one propagating DoF, as it happens in flat space. As we mentioned above, the fact that AdS gauge potentials with non rectangular Young tableaux carry more DoF's than corresponding flat space potentials might be the origin of this difficulty in integrating the curvatures $R^{I_{1} \ldots I_{n}}$ for $n \neq 0, s .{ }^{9}$ Indeed, the light cone analysis of section 4.1 shows that the whole sum over fields $R^{I_{1} \ldots I_{n}}$ has the same degrees of freedom in both flat and AdS spaces. One can thus conclude that integrating $R^{I_{1} \ldots I_{n}}$ in AdS, if possible, would not give rise to the same gauge potentials as those introduced in flat space, namely $\varphi^{I_{1} \ldots I_{n}}$. We

\footnotetext{
${ }^{9}$ Remember that $R$ and $R^{I_{1} \ldots I_{s}}$ have rectangular Young tableaux and can thus be integrated.
} 
summarize the results for such spin-two case in the following table

\begin{tabular}{|c|ccc|}
\hline Curvature & $R \sim \square \square$ & $R^{I} \sim \square \square$ & $R^{I J} \sim \square \square$ \\
Potential & $\widetilde{\varphi} \sim \square$ & $\Downarrow$ \\
EoM & $G_{(2)}^{\mathrm{AdS}} \widetilde{\varphi}=0$ & $Q_{J} R^{I}=\operatorname{tr}^{J K} R^{I}=0$ & $G_{(2)}^{\mathrm{AdS}} \varphi^{I J}=0$ \\
$\operatorname{DoF}$ & 0 & 1 & \\
\hline
\end{tabular}

The total number of DoF's is two, just like those of a massive spin-two in $D=3$.

\section{Conclusions}

We have constructed a relativistic action for a massive particle with higher spin by dimensionally reducing a massless model. ${ }^{10}$ The massless model used, the spinning particle with local $\mathrm{SO}(N)$ extended susy on the worldline, propagates degrees of freedom only in a spacetime of even dimension, so that the emerging model lives in a spacetime of odd dimensions. We have covariantized it to introduce a coupling to (A)dS spaces, and shown that the physical degrees of freedom propagating at the quantum level satisfy the FierzPauli equations extended to (A)dS spaces. The massless limit of the model contains the same number of degrees of freedom. Its covariant description, arising for the quantum Dirac constraints related to the gauge symmetries of the particle action, has a geometric interpretation in terms of curvatures, but a reformulation in terms of gauge potentials is generically more complex that the one arising in flat space, and we have just presented the simple example of $s=2$ in $D=3$. We have not produced a general analysis of the massless case to uncover if and how the (A)dS geometrical equations are related to massless gauge potentials. Indeed, it is also conceivable that some of the degrees of freedom could be realized in the form of "partially massless states" discovered in [41, 42], and further analyzed in [43], where the authors derived the generating function of HS (A)dS actions for both massive and partially massless fields by applying the log radial reduction technique [44] to a one-dimension higher massless HS theory, an idea that was further developed within the tractor and BRST set up in [45]. It could be interesting to see if and how partially massless states can be described in first quantization.

One could proceed further in the first quantized description of our model by considering a closed worldline, so to analyze the corresponding one loop effective action. Path integrals on curved spaces need a regularization [46], but they can be used successfully in worldline approaches to QFT problems [47]. We expect that for the present model the counterterms

\footnotetext{
${ }^{10}$ Similarly, dimensional reduction has been used to find actions for massive HS fields, as for example in refs $[39,40]$.
} 
identified in [48] are enough for carrying out the perturbative evaluation of the one loop effective action. One might also expect that an exact evaluation be possible, as similar result have been found on AdS spaces for higher spin fields [49, 50].

Another direction where to extend the present work is to consider dimensional reduction in more than one dimension. This may allow to find worldline actions that describe propagation of several multiplets of massive and massless HS excitations in flat and AdS spaces. Of course, it would be extremely interesting to see how to make the various HS particles self-interact in a first quantized picture, though this is a sensibly harder problem.

\section{Acknowledgments}

The authors are grateful to P. Sundell for discussions. They acknowledge the partial support of UCMEXUS-CONACYT grant CN-12-564. RB thanks the Universidad Andrés Bello for hospitality. EL acknowledges partial support of SNF Grant No. 200020-149150/1.

Open Access. This article is distributed under the terms of the Creative Commons Attribution License (CC-BY 4.0), which permits any use, distribution and reproduction in any medium, provided the original author(s) and source are credited.

\section{References}

[1] M.A. Vasiliev, Consistent equation for interacting gauge fields of all spins in $(3+1)$-dimensions, Phys. Lett. B 243 (1990) 378 [INSPIRE].

[2] M.A. Vasiliev, Higher spin gauge theories in four-dimensions, three-dimensions and two-dimensions, Int. J. Mod. Phys. D 5 (1996) 763 [hep-th/9611024] [INSPIRE].

[3] M.A. Vasiliev, Nonlinear equations for symmetric massless higher spin fields in (A)d $S_{d}$, Phys. Lett. B 567 (2003) 139 [hep-th/0304049] [INSPIRE].

[4] E. Sezgin and P. Sundell, Massless higher spins and holography, Nucl. Phys. B 644 (2002) 303 [Erratum ibid. B 660 (2003) 403] [hep-th/0205131] [INSPIRE].

[5] I.R. Klebanov and A.M. Polyakov, AdS dual of the critical $O(N)$ vector model, Phys. Lett. B 550 (2002) 213 [hep-th/0210114] [INSPIRE].

[6] E. Sezgin and P. Sundell, Holography in $4 D$ (super) higher spin theories and a test via cubic scalar couplings, JHEP 07 (2005) 044 [hep-th/0305040] [INSPIRE].

[7] S. Giombi and X. Yin, Higher spin gauge theory and holography: the three-point functions, JHEP 09 (2010) 115 [arXiv:0912.3462] [INSPIRE].

[8] M.R. Gaberdiel and R. Gopakumar, An AdS $S_{3}$ dual for minimal model CFTs, Phys. Rev. D 83 (2011) 066007 [arXiv: 1011.2986] [INSPIRE].

[9] V.E. Didenko and E.D. Skvortsov, Exact higher-spin symmetry in CFT: all correlators in unbroken Vasiliev theory, JHEP 04 (2013) 158 [arXiv: 1210.7963] [INSPIRE].

[10] S. Giombi and I.R. Klebanov, One loop tests of higher spin AdS/CFT, JHEP 12 (2013) 068 [arXiv: 1308.2337] [INSPIRE].

[11] X. Bekaert, S. Cnockaert, C. Iazeolla and M.A. Vasiliev, Nonlinear higher spin theories in various dimensions, hep-th/0503128 [INSPIRE]. 
[12] X. Bekaert, N. Boulanger and P. Sundell, How higher-spin gravity surpasses the spin two barrier: no-go theorems versus yes-go examples, Rev. Mod. Phys. 84 (2012) 987 [arXiv: 1007.0435] [INSPIRE].

[13] A. Sagnotti, Notes on strings and higher spins, J. Phys. A 46 (2013) 214006 [arXiv:1112.4285] [INSPIRE].

[14] V.E. Didenko and E.D. Skvortsov, Elements of Vasiliev theory, arXiv:1401.2975 [INSPIRE].

[15] M.A. Vasiliev, Higher-spin theory and space-time metamorphoses, arXiv:1404.1948 [INSPIRE].

[16] F. Bastianelli, O. Corradini and E. Latini, Higher spin fields from a worldline perspective, JHEP 02 (2007) 072 [hep-th/0701055] [INSPIRE].

[17] F. Bastianelli, O. Corradini and E. Latini, Spinning particles and higher spin fields on (A)dS backgrounds, JHEP 11 (2008) 054 [arXiv: 0810.0188] [INSPIRE].

[18] F. Bastianelli, R. Bonezzi, O. Corradini and E. Latini, Effective action for higher spin fields on (A)dS backgrounds, JHEP 12 (2012) 113 [arXiv:1210.4649] [INSPIRE].

[19] V.D. Gershun and V.I. Tkach, Classical and quantum dynamics of particles with arbitrary spin, JETP Lett. 29 (1979) 288 [Pisma Zh. Eksp. Teor. Fiz. 29 (1979) 320] [InSPIRE].

[20] P.S. Howe, S. Penati, M. Pernici and P.K. Townsend, Wave equations for arbitrary spin from quantization of the extended supersymmetric spinning particle, Phys. Lett. B 215 (1988) 555 [INSPIRE].

[21] P.S. Howe, S. Penati, M. Pernici and P.K. Townsend, A particle mechanics description of antisymmetric tensor fields, Class. Quant. Grav. 6 (1989) 1125 [INSPIRE].

[22] W. Siegel, Conformal invariance of extended spinning particle mechanics, Int. J. Mod. Phys. A 3 (1988) 2713 [INSPIRE].

[23] W. Siegel, All free conformal representations in all dimensions, Int. J. Mod. Phys. A 4 (1989) 2015 [INSPIRE].

[24] S.M. Kuzenko and Z. Yarevskaya, Conformal invariance, $N$-extended supersymmetry and massless spinning particles in anti-de Sitter space, Mod. Phys. Lett. A 11 (1996) 1653 [hep-th/9512115] [INSPIRE].

[25] V. Bargmann and E.P. Wigner, Group theoretical discussion of relativistic wave equations, Proc. Nat. Acad. Sci. 34 (1948) 211 [inSPIRE].

[26] X. Bekaert and N. Boulanger, Tensor gauge fields in arbitrary representations of $G L(D, R)$ : duality and Poincaré lemma, Commun. Math. Phys. 245 (2004) 27 [hep-th/0208058] [INSPIRE].

[27] X. Bekaert and N. Boulanger, Tensor gauge fields in arbitrary representations of $G L(D, R)$. II. Quadratic actions, Commun. Math. Phys. 271 (2007) 723 [hep-th/0606198] [INSPIRE].

[28] C. Fronsdal, Massless fields with integer spin, Phys. Rev. D 18 (1978) 3624 [inSPIRE].

[29] J.M.F. Labastida, Massless particles in arbitrary representations of the Lorentz group, Nucl. Phys. B 322 (1989) 185 [INSPIRE].

[30] D. Francia and A. Sagnotti, On the geometry of higher spin gauge fields, Class. Quant. Grav. 20 (2003) S473 [Comment. Phys. Math. Soc. Sci. Fenn. 166 (2004) 165] [PoS (JHW2003) 005] [hep-th/0212185] [INSPIRE].

[31] A. Sagnotti and M. Tsulaia, On higher spins and the tensionless limit of string theory, Nucl. Phys. B 682 (2004) 83 [hep-th/0311257] [INSPIRE]. 
[32] I. Bandos, X. Bekaert, J.A. de Azcarraga, D. Sorokin and M. Tsulaia, Dynamics of higher spin fields and tensorial space, JHEP 05 (2005) 031 [hep-th/0501113] [INSPIRE].

[33] J. Scherk and J.H. Schwarz, How to get masses from extra dimensions, Nucl. Phys. B 153 (1979) 61 [INSPIRE].

[34] M. Fierz and W. Pauli, On relativistic wave equations for particles of arbitrary spin in an electromagnetic field, Proc. Roy. Soc. Lond. A 173 (1939) 211 [inSPIRE].

[35] R.R. Metsaev, Light cone form of field dynamics in anti-de Sitter space-time and AdS/CFT correspondence, Nucl. Phys. B 563 (1999) 295 [hep-th/9906217] [INSPIRE].

[36] L. Brink, R.R. Metsaev and M.A. Vasiliev, How massless are massless fields in AdS $S_{d}$, Nucl. Phys. B 586 (2000) 183 [hep-th/0005136] [INSPIRE].

[37] N. Boulanger, C. Iazeolla and P. Sundell, Unfolding mixed-symmetry fields in AdS and the BMV conjecture: I. General formalism, JHEP 07 (2009) 013 [arXiv:0812.3615] [INSPIRE].

[38] N. Boulanger, C. Iazeolla and P. Sundell, Unfolding mixed-symmetry fields in AdS and the BMV conjecture: II. Oscillator realization, JHEP 07 (2009) 014 [arXiv:0812.4438] [INSPIRE].

[39] I.L. Buchbinder and A. Reshetnyak, General Lagrangian formulation for higher spin fields with arbitrary index symmetry. I. Bosonic fields, Nucl. Phys. B 862 (2012) 270 [arXiv:1110.5044] [INSPIRE].

[40] A. Reshetnyak, General Lagrangian formulation for higher spin fields with arbitrary index symmetry. II. Fermionic fields, Nucl. Phys. B 869 (2013) 523 [arXiv:1211.1273] [InSPIRE].

[41] S. Deser and R.I. Nepomechie, Gauge invariance versus masslessness in de Sitter space, Annals Phys. 154 (1984) 396 [inSPIRE].

[42] S. Deser and A. Waldron, Partial masslessness of higher spins in (A)dS, Nucl. Phys. B 607 (2001) 577 [hep-th/0103198] [INSPIRE].

[43] K. Hallowell and A. Waldron, Constant curvature algebras and higher spin action generating functions, Nucl. Phys. B 724 (2005) 453 [hep-th/0505255] [INSPIRE].

[44] T. Biswas and W. Siegel, Radial dimensional reduction: anti-de Sitter theories from flat, JHEP 07 (2002) 005 [hep-th/0203115] [INSPIRE].

[45] M. Grigoriev and A. Waldron, Massive higher spins from BRST and tractors, Nucl. Phys. B 853 (2011) 291 [arXiv: 1104.4994] [INSPIRE].

[46] F. Bastianelli and P. van Nieuwenhuizen, Path integrals and anomalies in curved space, Cambridge University Press, Cambridge U.K. (2006) [INSPIRE].

[47] F. Bastianelli and A. Zirotti, Worldline formalism in a gravitational background, Nucl. Phys. B 642 (2002) 372 [hep-th/0205182] [INSPIRE].

[48] F. Bastianelli, R. Bonezzi, O. Corradini and E. Latini, Extended SUSY quantum mechanics: transition amplitudes and path integrals, JHEP 06 (2011) 023 [arXiv:1103.3993] [INSPIRE].

[49] R. Camporesi and A. Higuchi, Arbitrary spin effective potentials in anti-de Sitter space-time, Phys. Rev. D 47 (1993) 3339 [inSPIRE].

[50] R. Camporesi and A. Higuchi, Spectral functions and zeta functions in hyperbolic spaces, $J$. Math. Phys. 35 (1994) 4217 [INSPIRE]. 